\title{
Analysis of cellular fatty acids and phenotypic relationships of Agrobacterium, Bradyrhizobium, Mesorhizobium, Rhizobium and Sinorhizobium species using the Sherlock Microbial Identification System
}

\author{
S. W. Tighe, ${ }^{1} \dagger$ P. de Lajudie, ${ }^{2,3}$ K. Dipietro, ${ }^{1}$ K. Lindström, ${ }^{4}$ G. Nick ${ }^{4}$ \\ and B. D. W. Jarvis ${ }^{5}$
} Author for correspondence: S. W. Tighe. Tel: +1 802372 4104. Fax: + 18028786765 .
e-mail: stighe@globalnetisp.net

1 Analytical Services, Inc., PO Box 515, Williston, VT, USA

2 Laboratoire de Symbioses Tropicales et

Mediterraneennes, IRD, Campus de Baillarguet, BP 5035, Montpellier Cedex, France

3 Laboratorium voor Microbiologie, Universiteit Gent, K. L.

Ledeganckstraat 35, B-9000 Ghent, Belgium

4 Department of Applied Chemistry and Microbiology, University of Helsinki, FIN-00014, Helsinki, Finland

5 Rivendell, Old West Road, Palmerston North, RD4, New Zealand
Previous studies have demonstrated that cellular fatty acid analysis is a useful tool for identifying unknown strains of rhizobia and establishing taxonomic relationships between the species. In this study, the fatty acid profiles of over 600 strains belonging to the genera Agrobacterium, Bradyrhizobium, Mesorhizobium, Rhizobium and Sinorhizobium were evaluated using the gaschromatography-based Sherlock Microbial Identification System (MIS). Data collected with the MIS showed that the three phylogenetically defined biovars of the genus Agrobacterium formed discrete clusters, whilst species belonging to the genus Mesorhizobium formed three subclusters which were easily distinguished. These three subclusters contained Mesorhizobium ciceri and Mesorhizobium mediterraneum, Mesorhizobium tianshanense fatty acid group I and Mesorhizobium plurifarium, and Mesorhizobium huakuii and Mesorhizobium loti. The genus Sinorhizobium was composed of an individual position for Sinorhizobium meliloti and a large cluster comprising Sinorhizobium fredii, Sinorhizobium saheli, Sinorhizobium terangae, Sinorhizobium kostiense and Sinorhizobium arboris. S. meliloti contained significantly higher levels of the fatty acid 19:0 cyclo $\omega 8$ cis and clustered with Rhizobium sp. (Hedysarum coronarium). However, discrimination between the species of genera Sinorhizobium and Rhizobium was a function of the concentration of 16:0 3-OH. The genus Rhizobium contained a single cluster containing Rhizobium sp. (Hedysarum coronarium), Rhizobium gallicum, Rhizobium leguminosarum and Rhizobium etli, along with individual positions for Rhizobium giardinii, Rhizobium tropici, Rhizobium galegae and Rhizobium hainanense. $R$. tropici and $R$. hainanense exhibited similarity to Agrobacterium biovar 2, whilst $R$. galegae was similar to Agrobacterium biovar 1. $R$. giardinii appeared unique, with comparatively little similarity to the other species. Analysis of the genus Bradyrhizobium revealed large differences from the other genera studied. Two subgroups of Bradyrhizobium elkanii were detected and easily distinguished from Bradyrhizobium japonicum. Bradyrhizobium liaoningense and Bradyrhizobium sp. (Arachis hypogaea), a group isolated from Chinese peanut plants, showed similarities to $B$. japonicum, whilst a subgroup of $M$. tianshanense appeared identical to Bradyrhizobium sp. (Arachis hypogaea).

Keywords: Rhizobium taxonomy, fatty acid analysis, Sherlock Microbial Identification System

†Present address: PO Box 24, Grand Isle, VT 05458, USA.

Abbreviations: $E C L$, equivalent chain length; MIS, Microbial Identification System. 


\section{INTRODUCTION}

Organisms collectively known as rhizobia are important plant-associated bacteria which have been studied extensively over the past twenty years. As the taxonomy of the rhizobia changes and additional species are described, the need for rapid comparisons and identification is necessary. In this study, we have characterized the cellular fatty acids of over 600 strains of rhizobia using the gas-chromatography-based Sherlock Microbial Identification System (MIS). This system is designed to analyse bacterial extracts for cellular fatty acids in an automated approach, and has been shown to be very sensitive and reproducible (Sasser, 1990). It was employed in this study because of the MIS software's library generation capability, the large number of known compounds in the system's peak naming table library, the ability to compare a large number of strains over a period of time, and the routine use of this system for bacterial analysis in many laboratories.

Phylogenetic data place the rhizobia and agrobacteria in the $\alpha$-subdivision of the subclass Proteobacteria, and divide them into seven genera: Rhizobium, Agrobacterium, Sinorhizobium, Mesorhizobium, Allorhizobium, Azorhizobium and Bradyrhizobium. Members of the genera Rhizobium and Sinorhizobium are significant because of their ability to nodulate the roots of leguminous plants and carry out nitrogen fixation (Keyser et al., 1982; Long, 1989; Lynch, 1976). Although this is also true of the genus Bradyrhizobium, their in vitro cellular proliferation rate is slower than that of the genus Rhizobium and they are genetically different from the other rhizobia (Jordan, 1982). Currently, there are four recognized species belonging to the genus Rhizobium which have been validated using polyphasic taxonomy; these are Rhizobium etli, Rhizobium galegae, Rhizobium leguminosarum and Rhizobium tropici. Additional studies have revealed that six additional species exist, these include Rhizobium hainanense (Chen et al., 1997), Rhizobium huautlense (Wang et al., 1998), Rhizobium giardinii (Phaseolus genomic species 2 type H152), Rhizobium gallicum (Phaseolus genomic species 1 type R602) (Amarger et al., 1997), Rhizobium mongolense (Van Berkum et al., 1998) and possibly Rhizobium sp. (Hedysarum coronarium) (Casella et al., 1986) although this organism has not yet been validated.

Recent changes to the genus Rhizobium include the transfer of Rhizobium loti and Rhizobium huakuii to the new genus Mesorhizobium. The genus Mesorhizobium was proposed to accommodate the meso-growing rhizobia related to $R$. loti . The current species belonging to this genus include Mesorhizobium loti, Mesorhizobium huakuii, Mesorhizobium mediterraneum, Mesorhizobium ciceri, Mesorhizobium tianshanense (Jarvis et al., 1997), Mesorhizobium plurifarium (de Lajudie et al., 1998b) and Mesorhizobium amorphae (Wang et al., 1999).

In 1988 the genus Sinorhizobium was proposed to accommodate fast-growing nodule-forming soybean rhizobia (Chen et al., 1988) and until recently contained only Sinorhizobium fredii, Sinorhizobium xinjiangense (Chen et al., 1988), and Sinorhizobium meliloti. However, over the past several years, this genus has expanded to include Sinorhizobium terangae and Sinorhizobium saheli (de Lajudie et al., 1994), Sinorhizobium kostiense and Sinorhizobium arboris (Nick et al., 1999) and Sinorhizobium medicae (Rome et al., 1996), Although $S$. xinjiangense was described some time ago, it is controversial due to the lack of polyphasic data and close similarity to $S$. fredii (Jarvis et al., 1992). In this study, we did not evaluate strains of $S$. medicae or $S$. xinjiangense.

Unlike the genera Rhizobium, Sinorhizobium, Mesorhizobium, Bradyrhizobium, Allorhizobium and Azorhizobium, members of the genus Agrobacterium are not capable of nodulating the roots of leguminous plants, but instead are considered plant pathogens capable of causing gall disease and root hair disease (Bradbury, 1986; Jordan, 1984). Species belonging to this genus were previously separated based on phytopathogenicity data and included Agrobacterium tumefaciens, Agrobacterium radiobacter, Agrobacterium rhizogenes, Agrobacterium rubi and Agrobacterium vitis. More recent work indicates that classification should be based on the three phylogenetically defined biovars rather than phytopathogenicity (Willems \& Collins, 1993). This proposal has recognized biovar 1,2 and 3 as $A$. tumefaciens, $A$. rhizogenes and $A$. vitis, respectively. Cellular fatty acid and phylogenetic data are in agreement and support the biovar classification (Jarvis et al., 1996; Sawada et al., 1992; Yokota et al., 1993).

The taxonomy of the genus Bradyrhizobium has not experienced the rapid changes that have occurred in other genera. Bradyrhizobium japonicum and Bradyrhizobium elkanii have been established species for some time (Young \& Haukka, 1996), but recent studies have proposed a new species, Bradyrhizobium liaoningense (Xu et al., 1995). B. liaoningense was proposed for extra-slow-growing bradyrhizobia which exhibited unique phenotypic and genotypic characteristics. Studies performed on isolates recovered from the root nodules of peanuts (Arachis hypogaea) have also been investigated (Zhang et al., 1999) and are presented in this manuscript for purposes of comparison with the other species. Although the genus Bradyrhizobium is not considered a close relative of other rhizobia, it was included in this study because (1) a large number of strains were analysed by methods identical to those used in the analysis of the other rhizobia, (2) several unique clusters were detected, and (3) results indicated that several strains of $M$. tianshanense appeared to belong to the genus Bradyrhizobium.

Also included among the rhizobia are the genera Azorhizobium, which contains the single species Azorhizobium caulinodans, and Allorhizobium which 
contains the single species Allorhizobium undicola (de Lajudie et al., 1998a). These organisms, however, were not evaluated in this study and data are not presented.

\section{METHODS}

Bacterial strains and growth conditions. All strains analysed in this study were obtained from established culture collections and had been previously characterized using phylogenetic techniques, metabolic characterization, protein gel electrophoresis, DNA-DNA hybridization and/or numerical analysis. All strains were grown using methods previously described (Graham et al., 1995; Jarvis \& Tighe, 1994).

All strains belonging to the genus Agrobacterium were received from the Culture Collection of the Laboratorium voor Microbiologie, Ghent, Belgium (LMG). Agrobacterium biovar 1 strains were LMG 187t, LMG 196, LMG 317, LMG 26, LMG 64, LMG 147, LMG 228, LMG 14, LMG 296T1t, LMG 176, LMG 340, LMG 305, LMG 31, LMG 139, LMG 140, LMG 200, LMG 226, LMG 27, LMG 201, LMG 225, LMG 296T2, LMG 146, LMG 198, LMG 15, LMG 181, LMG 290, LMG 267, LMG 232, LMG 62, LMG 148, LMG 338, LMG 177, LMG 186 and LMG 142. Agrobacterium biovar 2 strains were LMG 155, LMG 231, LMG 219, LMG 150, LMG 168, LMG 229, LMG 162, LMG 149, LMG 161, LMG 152, LMG 172, LMG 154, LMG 341, LMG 307, LMG 253 and LMG 63. Agrobacterium biovar 3 strains were LMG 257, LMG 8749, LMG 8750T, LMG 260, LMG 256, LMG 258, LMG 259, LMG 261, LMG 262 and LMG 8748.

All strains of B. japonicum and B. elkanii were received from the University of Minnesota (U of $M$ ) Soil Sciences Department. B. elkanii strains were UMR 40 (USDA 61), UMR 223 (IRC353C), UMR 130 (USDA 130), UMR 224 (IRC353B1), UMR 96 (S5), UMR 127 (BTIRC20), UMR 48 (USDA 31), UMR 128 (BT-2177A), NA6734, NA6742, NA6726, NA6733, CIAT 2335, NA6741, CK127, CK115, CK121, CK119, UMR 15 (USDA 40), NA6705, CK1, NA6710, NA6711, NA6708, CB3097, NA6728 and NA6809. B. japonicum strains were UMR 12 (USDA 126), NA6407, UMR 115 (USDA 115), UMR 90 (WB69), UMR 230 (USDA 141), UMR 23 (CB1809), UMR 25 (USDA 140), UMR 110 (USDA 110), UMR 6 (RCR3425), UMR 92 (CB2966), UMR 88 (CB2811), UMR 39 (USDA 125), UMR 62 (USDA 38), UMR 16 (USDA 62), UMR 21 (USDA 44), UMR 123 (USDA 123), NA6471, USDA 129, TAL1812, CK215, TAL1905, TAL1843, NA6721, NA6460, CK143, CK147, 1188, 1189, CB3053, CB283, WU253, CIAT 3144, USDA 454, USDA 467, CIAT 4103, CIAT 4967, CIAT 4100, CIAT 4099, CIAT 861, USDA 450, USDA 469, CIAT 3649, CIAT 3918 and CIAT 2434. The strains of $B$. liaoningense received from the Soil and Fertilizers Institute (SFI), CAAS, Beijing, Peoples' Republic of China, were 2043, 2064, 2061, 2068, 2281, DE544, 2062 and 2309. Strains of Bradyrhizobium sp. (Arachis hypogaea) were received from Sichuan Agricultural University, Peoples' Republic of China and the University of Helsinki, Department of Applied Chemistry and Microbiology. Helsinki, Finland (HAMBI). Strains were Spr 3-7, Spr 7-8, Spr 4-2, Spr 63, Spr 3-5, Spr 2-9, Spr 2-8, Spr 7-9, Spr 3-6, Spr 7-5, Spr 41, Spr 3-4, Spr 4-4, Spr 4-6, Spr 3-2, Spr 4-5, Spr 7-7, Spr 710, Spr 3-1, Spr 7-1, Spr 3-3 and Spr 4-10.

Strains of $M$. ciceri received from HAMBI were 522, IC 2091, UPM Ca116, 3H0a10, CP77, IC2018, UPM Ca7t (ATCC 51585), CP92 and CP76. Strains of M. plurifarium received from the Institut de Recherche pour le Developpement (IRD) Dakar, Senegal, were ORS 1024, ORS 1026, ORS 1036, ORS 1032, ORS 1038, ORS 1015, ORS 1035, ORS 1030, ORS 1031, ORS 1029, ORS 1037, ORS 1010, ORS 1040, ORS 1001, ORS 654-204, ORS 656205 and ORS 1018. Strains of Mesorhizobium huakuii received from the Culture Collection of Beijing Agricultural University (CCBAU), Peoples' Republic of China, were CBAU2609t, B23-106-26, 7034, T2, 38, 7034, B1940, 31, 9109, 9124, 7653, 9104, RA01, RA05, RA04 and SR98. Strains of $M$. lot $i$ were received from the American Type Culture Collection (ATCC) Manassas, VA, USA, and the Massey University Culture Collection (MUCC), Palmerston North, New Zealand, and were NZP2048, NZP2079, ATCC 33669, NZP2196 (SU343), NZP2048, NZP2227 (461), NZP2298 (L72M103c), NZP2037, NZP2230 (CC809a), NZP2235 (CC812a), NZP2146, NZP2234 (CC811), NZP2300, (L72M115c), NZP2241, NZP2203, NZP2195 and NZP2014. Strains of $M$. mediterraneum received from the Universite Claude Bernard-Lyon I (UCBL) Villeurbanne Cedex, France, were CP12, CP54, CP18, IC60, CP8, UPM Ca36t, CP14, Ca7, IC72M (ATCC 51586), CP28, H45 and CP24. Strains of Mesorhizobium tianshanense group I received from CCBAU were 005B, A-1-BS, 91x10, A6, 016Bm, 91x05, G2 and 91x11. Strains of $M$. tianshanense group II received from CCBAU were 91x13, 91x72, 91x07 and $91 \times 09$.

$R$. etli strains received from the Centro de Investigacion sobre Fijacion de Nitrogeno (CIFN), Cuernavaca, Morales, Mexico were F8, F17, F14, TAL182, 127K105, NITRAGIN 8251, VIK, CFN1, BRA5, 1G2, 2CH3, 2G18, 2G15, 2G11, MM13, 1CH9, 2M21, 1M11, 2G15, 2M20 and 1M18. Strains received from Institut National de la Recherche Agronomique (INRA) Dijon Cedex, France were BC45 (MSDJ 2291), BB13 (MSDJ 2249), BA12 (MSDJ 2223), CP115 (MSDJ 2531), GD112 (MSDJ 2398), BC15 (MSDJ 2277), CP134 (MSDJ 2535), CP91 (MSDJ 2488) and GD164 (MSDJ 2414). R. galegae strains received from HAMBI were 1460, 54013, 1428, 1145, 1146, 1143, 1141, 490, 1189, $1147,1151,1184,1183,1174,1122,1461,1186,503$ and 1185. R. gallicum strains received from INRA were JSJ45, FI27, PHP222 (MSDJ 1100), R602 (MSDJ 1081), DC22 (MSDJ 114), PHD12 (MSDJ 960), JSJ18, JSJ42, JSJ55, JSJ15, 118H1，JSJ46，PHP18，Sm2，JSJ89，116A15 and JSJ560. Strains of $R$. giardinii received from INRA were JSJRt27, Ro67 (MSDJ 67), Ro84 (MSDJ 84), H236 (MSDJ 498), H231 (MSDJ 493), Ro70 (MSDJ 70), H251 (MSDJ 505), H152 (MSDJ 444), Ro17 (MSDJ 17), Ro7 (MSDJ 7), PHF28 (MSDJ 1084), Ro68 (MSDJ 68), Ro52 (MSDJ 52), H153 (MSDJ 447), Ro75 (MSDJ 75), Ro53 (MSDJ 53), Ro61 (MSDJ 61), H241 (MSDJ 499), H325 (MSDJ 707) and JSJ3. $R$. hainanense strains received from the CCBAU were I66, S25, I27, H14, I65, I33, I36, I20, I54, I2, I32, I64 and I12. Rhizobium sp. (Hedysarum coronarium) strains received from the Universita Degli Studi di Padova (UDSP), Padova, Italy were RHF, IS123, RH44, HCNT1, RH19, A10, 835, S6, RH100, MS19, RHAL, VIL3-1, 30-21, VIL2, CC1337, VIL3-2, HA7, 47 and HCNA. R. leguminosarum strains received from MUCC were F310 (MU367), NZP1/6 (MU224), K8 (MU258), F300 (MU362), NZP5459 (MU273), M1928 (?), B248 (?), NZP5262 (CB596, MU272), NZP540 (SU39, MU269), SP18, NZP5230 (TA101, MU268), NZP5225 (MU267), TA1 (MU237), NZP514 (MU231), MU252, NZP5479 and MU632. Strains received from the ATCC were ATCC 14479 and ATCC 14480. Strains received from INRA were H242 (MSDJ 500), H136 
(MSDJ 434), PhC152 (MSDJ 1083), Ro34 (MSDJ 34), PhF32 (MSDJ 1102), Ro29 (MSDJ 29), PhP17 (MSDJ 961), Ro35 (MSDJ 35) and H123 (MSDJ 429). R. tropici strains received from the CIFN were BR859, 10043, BR845, BR836, BR847, BR842, BR864, CFN299, BR863, BR10042, BR862, BR857, BR846, CIAT 899t, C-05-I, BR852, BR850 and BR853. Strains received from the INRA were CESTAS 221(MSDJ 2932), CESTAS 114 (MSDJ 2870), CARA 56 (MSDJ 4573), AIREL 35 (MSDJ 4571), CESTAS 134 (MSDJ 2873), AIREL 27 (MSDJ 4569), AIREL 28 (MSDJ 4452) and BA45 (MSDJ 2241).

S. fredii strains received from MUCC were MU640 (USDA 217), MU646 (USDA 257), MU636 (USDA 191), MU643 (USDA 192), MU637 (USDA 194) and MU638 (USDA 298). Strains received from the CCBAU were CCBAU 116 , CCBAU 110, CCBAU 112, CCBAU 105, CCBAU 103, CCBAU 115 and CCBAU 169. Strains received from the John Innes Institute, Norwich, UK were USDA 193, USDA 205t, USDA 201, USDA 206 and USDA 214. S. meliloti strains received from the MUCC were MU462 (U45), MU442 (CC2017), MU463 (300a13), MU461 (PDDCC1322), MU464 (Balsac), MU443 (CB1170), MU663 (2011) and MU441 (SU47). Strains received from Pennsylvania State University, PA, USA were M161, CC2003, 102FSI, 74B12, M275, 15A6, CC2013, M205, U102 and M1. Strains received from the ATCC were ATCC 10310 and ATCC 9930. S. saheli strains received from HAMBI were 1496 and 1495. Strains received from IRD were ORS 609t, ORS 611, ORS 600 and ORS 12. S. terangae strains received from IRD were ORS 1057, ORS 1071, ORS 1007 , ORS 1013, ORS 1058, ORS 22, ORS 53, ORS 1009, ORS 604, ORS 51, ORS 52, ORS 1047, ORS 1025, ORS 1016, ORS 1073 and ORS 613. S. kostiense strains received from HAMBI were 1492, 1491, 1496, 1498, 1481, 1486, 1493, 1504, 1501, 1485, 1502, 1505, 1489, 1484, 1488, 1483, 1490, 1482 and 1679. S. arboris strains received from HAMBI were 1680, 1704, 1707, 1396, 1706, 1685, 1552, 1708 and 1700.

Analysis of cellular fatty acids. Harvesting and extraction procedures were performed according to methods described by Sasser (1990). Fatty acid analysis was performed using a Hewlett Packard 5890 series II gas chromatograph equipped with an Ultra2 capillary column. Construction of the microbial library and similarity data were accomplished using the MIS Sherlock software (Microbial ID). Threedimensional plotting of principal components was performed using standard computer automated design software using coordinates from the MIS 2-D principal component plotting data.

The fatty acid compounds for each strain were identified by first converting the total cellular fatty acids to fatty acid methyl esters. Although some research has shown that the Sherlock MIS extraction method for converting cellular fatty acids to the fatty acid methyl ester derivatives may produce artifacts for rhizobia isolates (Orgambide et al., 1993), the technique was performed throughout our research to preserve the standard operating procedure recommended by the Sherlock MIS suppliers. Identification of each fatty acid compound was determined using gas chromatography followed by data analysis with the Sherlock MIS software. Cellular fatty acids were identified by comparing the equivalent chain length (ECL) of each compound to a peak naming table that contains over 115 known standards. The quantity of each compound in a strain was determined as a percentage of the total amount of fatty acid compounds present for that strain.

\section{RESULTS}

The MIS library generation software was used to produce the mean fatty acid profiles in Tables $1-4$. These tables indicate the mean percentage concentration of each compound detected in each species and were used by the MIS cluster analysis software to produce the coordinates in the principal component plot of Fig. 1. The cluster analysis software was further used to compute values presented in the similarity matrix using the identification values of all strains. Identification values are not included in this manuscript due to the large quantity of data they encompass.

In our previous work on the cellular fatty acids of rhizobia (Jarvis et al., 1996; Jarvis \& Tighe, 1994), several compounds were detected, but not successfully identified by the MIS software. Since that time, additional research has identified the compound

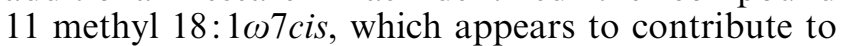
the discrimination of many species belonging to the genera Rhizobium and Mesorhizobium. Additionally, six new compounds were detected during this study from the new species examined. These compounds

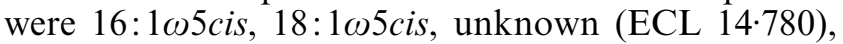
unknown (ECL 15.953), unknown (ECL 17.875), and unknown (ECL 18.794). It is important to note that when evaluating large groups of previously unexamined strains, the detection of new compounds is not uncommon and it is important for them to be included in current work for establishing accurate relationships between groups.

Although some of the new compounds encountered in this study have not yet been identified, they have been programmed into our MIS database as unknown compounds with the corresponding ECL value. Compounds that eluted from the chromatography column at essentially the same retention time were grouped into one feature known as a 'summed feature' by the Sherlock MIS software. Although this situation could in theory have been circumvented by the use of a longer, more efficient capillary column, it was not possible in practice because the entire MIS fatty acid peak naming table and data analysis software has been standardized to the current capillary column. Confirmation of fatty acid compounds using mass spectrometry or other methods was not attempted during this study and compound identification was solely determined by the MIS.

\section{Fatty acid composition}

The fatty acid compositions of the species analysed are shown in Tables 1-4. These tables depict all fatty acids detected at a mean concentration of greater than $0.01 \%$. Compounds in the table that are indicated with an asterisk were not used to produce the similarity matrix or 3-D principal component plot because they did not occur in all strains of the particular species and are not likely to be significant. Although insignificant concentrations of some compounds were detected, they were included in this manuscript to provide a 
Table 1. Mean fatty acid concentration of Agrobacterium and Bradyrhizobium species analysed in this study

Values are percentages of the total amount of fatty acid compounds present for that species. Numbers in parentheses indicate standard deviation.

\begin{tabular}{|c|c|c|c|c|c|c|c|c|}
\hline \multirow[t]{2}{*}{ Fatty acid compound } & \multicolumn{3}{|c|}{ Agrobacterium } & \multicolumn{5}{|c|}{ Bradyrhizobium } \\
\hline & Biovar 1 & Biovar 2 & Biovar 3 & $\begin{array}{l}\text { B. elkanii } \\
\text { group I }\end{array}$ & $\begin{array}{l}\text { B. elkanii } \\
\text { group II }\end{array}$ & B. japonicum & B. liaoningense & $\begin{array}{c}\text { Bradyrhizobium } \\
\text { sp. (Arachis) }\end{array}$ \\
\hline $10: 0$ & ND & $0.01(0.05)^{*}$ & ND & ND & ND & ND & ND & ND \\
\hline $10: 03-\mathrm{OH}$ & ND & $0 \cdot 24(0 \cdot 22)^{*}$ & ND & ND & ND & ND & ND & ND \\
\hline $11: 02-\mathrm{OH}$ & ND & ND & ND & ND & ND & ND & ND & ND \\
\hline Unknown (ECL 11.541) & $\mathrm{ND}$ & $0 \cdot 10(0 \cdot 11)^{*}$ & ND & $\mathrm{ND}$ & ND & $\mathrm{ND}$ & ND & ND \\
\hline Unknown (ECL 14.952) & ND & $0 \cdot 26(0 \cdot 16)^{*}$ & $0 \cdot 13(0 \cdot 22)^{*}$ & ND & ND & ND & ND & ND \\
\hline $12: 03-\mathrm{OH}$ & ND & ND & ND & $0.02(0 \cdot 08)^{*}$ & ND & $0.04(0.19)^{*}$ & ND & ND \\
\hline $13: 03-\mathrm{OH}$ & ND & ND & ND & ND & ND & $\mathrm{ND}$ & ND & ND \\
\hline $13: 1$ at $12-13$ & $0.49(0.21)$ & $0 \cdot 37(0 \cdot 10)$ & ND & $\mathrm{ND}$ & ND & $\mathrm{ND}$ & ND & ND \\
\hline $13: 0$ iso $3-\mathrm{OH}$ & ND & ND & ND & ND & ND & ND & ND & ND \\
\hline $14: 0$ & $0 \cdot 11(0 \cdot 12)^{*}$ & ND & ND & ND & ND & $0.01(0.07)^{*}$ & ND & ND \\
\hline Unknown (ECL 14·780) & $0.06(0 \cdot 11)^{*}$ & ND & $0 \cdot 22(0 \cdot 30)^{*}$ & ND & ND & ND & ND & ND \\
\hline $15: 0$ & $0.29(0 \cdot 19)^{*}$ & ND & ND & ND & ND & $0.04(0.15)^{*}$ & $0 \cdot 13(0 \cdot 36)^{*}$ & ND \\
\hline $15: 0$ anteiso & ND & ND & ND & ND & ND & ND & ND & ND \\
\hline $15: 0$ iso & ND & ND & ND & $\mathrm{ND}$ & ND & ND & ND & ND \\
\hline $15: 02-\mathrm{OH}$ & ND & ND & ND & ND & ND & ND & ND & ND \\
\hline $15: 03-\mathrm{OH}$ & $0.03(0.08)^{*}$ & ND & ND & ND & ND & ND & ND & ND \\
\hline $15: 0$ iso $3-\mathrm{OH}$ & ND & $4.02(0 \cdot 54)$ & ND & ND & ND & ND & ND & ND \\
\hline $15: 1 \omega 8 \mathrm{cis}$ & ND & ND & ND & ND & ND & ND & ND & ND \\
\hline Unknown (ECL 15.953) & $0 \cdot 27(0 \cdot 15)^{*}$ & $0 \cdot 28(0 \cdot 14)^{*}$ & ND & $\mathrm{ND}$ & ND & ND & ND & ND \\
\hline $16: 0$ & $9 \cdot 03(1.29)$ & $7 \cdot 14(1 \cdot 98)$ & $5.98(0.91)$ & $10 \cdot 49(2 \cdot 35)$ & $10 \cdot 36(0 \cdot 60)$ & $11.97(1.70)$ & $14 \cdot 83(1 \cdot 48)$ & $11 \cdot 14(1 \cdot 79)$ \\
\hline $16: 03-\mathrm{OH}$ & $4 \cdot 76(1 \cdot 23)$ & $5 \cdot 19(0 \cdot 88)$ & $2 \cdot 28(0 \cdot 78)$ & ND & ND & ND & ND & ND \\
\hline $16: 0$ iso & ND & ND & ND & ND & ND & ND & ND & ND \\
\hline $16: 1 \omega 5 \mathrm{cis}$ & ND & $\mathrm{ND}$ & ND & $0 \cdot 10(0 \cdot 27)^{*}$ & ND & $1.06(0.99)$ & $0 \cdot 88(0 \cdot 18)$ & $3.99(1.69)$ \\
\hline $17: 0$ & $0 \cdot 13(0 \cdot 14)^{*}$ & $0.06(0 \cdot 11)^{*}$ & ND & $0.03(0 \cdot 11)^{*}$ & ND & $0 \cdot 19(0 \cdot 31)^{*}$ & $0.79(1.06)$ & $0.03(0.13)^{*}$ \\
\hline $17: 0$ iso & ND & $0 \cdot 60(0 \cdot 16)$ & ND & ND & ND & ND & ND & ND \\
\hline $17: 0$ iso $3-\mathrm{OH}$ & ND & $0 \cdot 21(0 \cdot 18)^{*}$ & ND & $\mathrm{ND}$ & $\mathrm{ND}$ & $\mathrm{ND}$ & ND & ND \\
\hline $17: 0$ cyclo & $1.60(0.54)$ & $0 \cdot 74(0 \cdot 36)$ & ND & $0.95(0.48)$ & $0.50(0.53)^{*}$ & ND & ND & ND \\
\hline $17: 03-\mathrm{OH}$ & $0 \cdot 18(0 \cdot 18)^{*}$ & $\mathrm{ND}$ & ND & ND & ND & ND & ND & ND \\
\hline $17: 1 \omega 6 c i s$ & ND & ND & ND & ND & $0 \cdot 06(0 \cdot 22)^{*}$ & $0 \cdot 15(0 \cdot 29)^{*}$ & $0.66(0 \cdot 88)$ & $0.02(0.09)^{*}$ \\
\hline $17: 1 \omega 7 c i s$ & ND & ND & ND & ND & ND & ND & ND & $\mathrm{ND}$ \\
\hline $17: 1 \omega 8 \mathrm{cis}$ & $0 \cdot 22(0 \cdot 15)^{*}$ & ND & ND & $0.03(0.09)^{*}$ & $0.05(0 \cdot 17)^{*}$ & $0.48(0.52)$ & $0.99(1 \cdot 10)$ & $0.07(0 \cdot 17)^{*}$ \\
\hline Unknown (ECL 17·875) & ND & ND & ND & ND & ND & ND & ND & ND \\
\hline $18: 0$ & $0 \cdot 17(0.17)^{*}$ & $2.14(0.57)$ & $1 \cdot 21(0.48)$ & $0 \cdot 37(0 \cdot 29)$ & $0.15(0 \cdot 26)^{*}$ & $0 \cdot 62(0 \cdot 41)$ & $0.38(0.34)^{*}$ & $0.47(0.50)^{*}$ \\
\hline 11 methyl $18: 1 \omega 7 c i s$ & $0.23(0 \cdot 24)^{*}$ & $0 \cdot 14(0 \cdot 17)^{*}$ & $0.39(0 \cdot 36)^{*}$ & $0 \cdot 12(0 \cdot 31)^{*}$ & ND & $1 \cdot 91(3 \cdot 16)$ & $\mathrm{ND}$ & ND \\
\hline $18: 12-\mathrm{OH}$ & ND & $2 \cdot 22(0.87)$ & $1.29(0.43)$ & ND & ND & ND & ND & ND \\
\hline $18: 1 \omega 5 c i s$ & $\mathrm{ND}$ & ND & ND & $0.02(0 \cdot 08)^{*}$ & ND & $0.02(0 \cdot 09)^{*}$ & ND & ND \\
\hline $18: 1 \omega 9 \mathrm{cis}$ & ND & ND & ND & ND & ND & ND & ND & ND \\
\hline Unknown (ECL 18.794) & $0.01(0.03)^{*}$ & ND & ND & ND & ND & $0 \cdot 01(0 \cdot 08)^{*}$ & $0.27(0 \cdot 57)^{*}$ & ND \\
\hline $19: 0$ & ND & ND & ND & ND & ND & ND & ND & ND \\
\hline 19:0 cyclo $\omega 8$ cis & $18 \cdot 78(5 \cdot 14)$ & $34 \cdot 62(8.96)$ & $4 \cdot 11(2 \cdot 07)$ & $19.99(3.47)$ & $8 \cdot 82(2 \cdot 01)$ & $1 \cdot 16(1 \cdot 26)$ & $5 \cdot 04(2 \cdot 96)$ & $0.19(0.38)^{*}$ \\
\hline 19:0 10 methyl & $1.09(0 \cdot 30)$ & $0 \cdot 11(0 \cdot 18)^{*}$ & $2.03(0.67)$ & ND & ND & ND & $\mathrm{ND}$ & ND \\
\hline $20: 0$ & ND & ND & ND & ND & ND & ND & ND & ND \\
\hline $20: 1 \omega 9$ trans & ND & $0.04(0.09)^{*}$ & ND & ND & ND & $\mathrm{ND}$ & ND & ND \\
\hline $20: 2 \omega 6,9 c i s$ & $0.01(0.04)^{*}$ & $0 \cdot 13(0 \cdot 16)^{*}$ & ND & $0.29(0 \cdot 46)^{*}$ & ND & ND & $0 \cdot 11(0 \cdot 32)^{*}$ & ND \\
\hline $20: 3 \omega 6,9,12 c i s$ & $0 \cdot 33(0 \cdot 16)$ & $2 \cdot 46(0 \cdot 50)$ & $1.67(0 \cdot 38)$ & ND & ND & ND & $\mathrm{ND}$ & ND \\
\hline Summed feature $2 \dagger$ & $0.03(0.08)^{*}$ & ND & ND & ND & ND & ND & ND & ND \\
\hline Summed feature $3 \ddagger$ & $8 \cdot 15(1.90)$ & $3 \cdot 38(0 \cdot 44)$ & $7 \cdot 91(2 \cdot 57)$ & ND & ND & ND & ND & ND \\
\hline Summed feature $4 \S$ & $1.62(0 \cdot 40)$ & $0 \cdot 47(0 \cdot 19)$ & $5 \cdot 16(1 \cdot 16)$ & $0 \cdot 44(0 \cdot 38)$ & $0 \cdot 46(0 \cdot 42)$ & $1.06(0 \cdot 68)$ & $0.42(0.45)^{*}$ & $0.44(0.64)^{*}$ \\
\hline Summed feature $5 \|$ & ND & ND & ND & $0 \cdot 10(0 \cdot 35)^{*}$ & ND & ND & ND & ND \\
\hline Summed feature 7 & $52.41(6.86)$ & $35 \cdot 04(10 \cdot 78)$ & $67 \cdot 62(4 \cdot 85)$ & $67.05(5.04)$ & $79 \cdot 62(2 \cdot 55)$ & $81 \cdot 22(4 \cdot 66)$ & $75 \cdot 49(3.97)$ & $83.64(2.04)$ \\
\hline
\end{tabular}

ND, Not detected.

*Compounds not used for calculating identification values, similarity indices or principal component values.

$\dagger$ Summed feature $2=15: 1$ iso $\mathrm{H} / \mathrm{I}, 13: 03-\mathrm{OH}$.

\$ Summed feature $3=12: 0$ (aldehyde?), unknown (ECL 10.928), 16:1 iso I/14:0 3-OH.

$\S$ Summed feature $4=15: 0$ iso $2-\mathrm{OH}, 16: 1 \omega 7$ cis.

$\|$ Summed feature $5=17: 1$ iso/anteiso B.

- Summed feature $7=18: 1 \omega 7 \mathrm{cis} / \omega 9$ trans $/ \omega 12$ trans, $18: 1 \omega 7 \mathrm{cis} / \omega 9 \mathrm{cis} / \omega 12$ trans.

clearer representation of the fatty acids that could occur in each strain.

Agrobacterium. Fatty acid compounds detected in all biovars studied of the genus Agrobacterium included
16:0, 16:0 3-OH, 19:0 cyclo $\omega 8$ cis, 20:3 $\omega 6,9,12$ cis, summed feature 3, summed feature 4 and summed feature 7. Additional fatty acids detected in individual biovars included 13:1 at 12-13,17:0 cyclo and 19:0 10 methyl in Agrobacterium biovar 1; 13:1 at 12-13, 15:0 
Table 2. Mean fatty acid concentration of Mesorhizobium species analysed in this study

Values are percentages of the total amount of fatty acid compounds present for that species. Numbers in parentheses indicate standard deviation.

\begin{tabular}{|c|c|c|c|c|c|c|c|}
\hline Fatty acid compound & M. ciceri & M. huakuii & $\begin{array}{c}M . \\
\text { mediterraneum }\end{array}$ & M. loti & $\begin{array}{l}\text { M. tianshanense } \\
\text { group I }\end{array}$ & $\begin{array}{l}\text { M. tianshanense } \\
\text { group II }\end{array}$ & M. plurifarium \\
\hline $10: 0$ & ND & ND & ND & ND & ND & ND & ND \\
\hline $10: 03-\mathrm{OH}$ & ND & ND & ND & ND & ND & ND & $\mathrm{ND}$ \\
\hline $11: 02-\mathrm{OH}$ & $\mathrm{ND}$ & $\mathrm{ND}$ & ND & $\mathrm{ND}$ & ND & $\mathrm{ND}$ & $\mathrm{ND}$ \\
\hline Unknown (ECL 11.541) & ND & ND & $\mathrm{ND}$ & $\mathrm{ND}$ & $\mathrm{ND}$ & ND & $\mathrm{ND}$ \\
\hline Unknown (ECL 14.952) & ND & ND & ND & ND & ND & ND & ND \\
\hline $12: 03-\mathrm{OH}$ & $0.27(0 \cdot 22)^{*}$ & $0 \cdot 23(0 \cdot 31)^{*}$ & $0 \cdot 11(0 \cdot 16)^{*}$ & $0 \cdot 34(0 \cdot 36)^{*}$ & $0.09(0 \cdot 25)^{*}$ & ND & $0.20(0.25)^{*}$ \\
\hline $13: 03-\mathrm{OH}$ & ND & ND & ND & ND & ND & ND & ND \\
\hline $13: 1$ at $12-13$ & ND & ND & ND & ND & ND & ND & $\mathrm{ND}$ \\
\hline $13: 0$ iso $3-\mathrm{OH}$ & $0 \cdot 24(0 \cdot 23)^{*}$ & $1 \cdot 00(0 \cdot 26)$ & $0 \cdot 35(0 \cdot 28)^{*}$ & $1.23(0.35)$ & $0.27(0.38)^{*}$ & $\mathrm{ND}$ & $0.84(0.43)$ \\
\hline $14: 0$ & ND & ND & ND & ND & ND & ND & $\mathrm{ND}$ \\
\hline Unknown (ECL 14·780) & $0 \cdot 16(0 \cdot 25)^{*}$ & ND & $0 \cdot 04(0 \cdot 15)^{*}$ & ND & ND & ND & $0.01(0.06)^{*}$ \\
\hline $15: 0$ & $0.02(0.06)^{*}$ & ND & $0.04(0.13)^{*}$ & ND & ND & ND & ND \\
\hline $15: 0$ anteiso & ND & ND & ND & $0 \cdot 12(0 \cdot 51)^{*}$ & ND & ND & ND \\
\hline $15: 0$ iso & $0 \cdot 18(0 \cdot 23)^{*}$ & ND & $0 \cdot 13(0 \cdot 20)^{*}$ & $0 \cdot 13(0 \cdot 25)^{*}$ & $0 \cdot 16(0 \cdot 46)^{*}$ & ND & ND \\
\hline $15: 02-\mathrm{OH}$ & $\mathrm{ND}$ & $\mathrm{ND}$ & $\mathrm{ND}$ & $\mathrm{ND}$ & $\mathrm{ND}$ & $\mathrm{ND}$ & $\mathrm{ND}$ \\
\hline $15: 03-\mathrm{OH}$ & ND & $\mathrm{ND}$ & $\mathrm{ND}$ & ND & $\mathrm{ND}$ & ND & $\mathrm{ND}$ \\
\hline $15: 0$ iso $3-\mathrm{OH}$ & ND & ND & $\mathrm{ND}$ & $\mathrm{ND}$ & $\mathrm{ND}$ & ND & $\mathrm{ND}$ \\
\hline $15: 1 \omega 8$ cis & ND & ND & ND & $\mathrm{ND}$ & $\mathrm{ND}$ & ND & ND \\
\hline Unknown (ECL 15.953) & ND & ND & ND & ND & ND & ND & $\mathrm{ND}$ \\
\hline $16: 0$ & $13 \cdot 41(2 \cdot 11)$ & $15 \cdot 83(0 \cdot 98)$ & $10 \cdot 29(1 \cdot 39)$ & $14 \cdot 51(2 \cdot 15)$ & $12 \cdot 25(2 \cdot 26)$ & $11 \cdot 26(1 \cdot 11)$ & $11.60(1.53)$ \\
\hline $16: 03-\mathrm{OH}$ & ND & ND & ND & ND & $\mathrm{ND}$ & ND & ND \\
\hline $16: 0$ iso & ND & ND & ND & ND & ND & ND & $\mathrm{ND}$ \\
\hline $16: 1 \omega 5 \mathrm{cis}$ & ND & ND & ND & ND & $\mathrm{ND}$ & $2.65(0.53)$ & ND \\
\hline $17: 0$ & $1.29(0.95)$ & $0.42(0 \cdot 66)^{*}$ & $1.71(1.77)$ & $0.50(0.59)$ & $0.83(1.02)$ & $0.13(0 \cdot 26)^{*}$ & $0.69(0.51)$ \\
\hline $17: 0$ iso & $4.16(0.69)$ & $5.88(1.13)$ & $4 \cdot 19(1 \cdot 14)$ & $8 \cdot 17(1.93)$ & $3 \cdot 34(0 \cdot 68)$ & ND & $6.26(0.77)$ \\
\hline $17: 0$ iso $3-\mathrm{OH}$ & ND & ND & ND & ND & ND & $\mathrm{ND}$ & ND \\
\hline $17: 0$ cyclo & $0.84(0.38)$ & $0.70(0.52)$ & $0 \cdot 85(0 \cdot 82)$ & $0.69(0.51)$ & $0 \cdot 16(0 \cdot 31)^{*}$ & ND & $0.12(0.23)^{*}$ \\
\hline $17: 03-\mathrm{OH}$ & ND & ND & ND & ND & $\mathrm{ND}$ & ND & ND \\
\hline $17: 1 \omega 6 c i s$ & ND & ND & $\mathrm{ND}$ & ND & $0 \cdot 11(0 \cdot 32)^{*}$ & $0 \cdot 26(0 \cdot 30)^{*}$ & $0.09(0.26)^{*}$ \\
\hline $17: 1 \omega 7 c i s$ & $\mathrm{ND}$ & $\mathrm{ND}$ & $\mathrm{ND}$ & $\mathrm{ND}$ & $\mathrm{ND}$ & $\mathrm{ND}$ & ND \\
\hline $17: 1 \omega 8 c i s$ & $0 \cdot 15(0 \cdot 23)^{*}$ & $0.25(0 \cdot 40)^{*}$ & $0.51(0.65)^{*}$ & $0 \cdot 12(0 \cdot 31)^{*}$ & $0.52(0.77)^{*}$ & $0 \cdot 14(0 \cdot 27)^{*}$ & $0.71(0.50)$ \\
\hline Unknown (ECL 17.875) & $0.17(0.52)^{*}$ & ND & $0.50(0.99)^{*}$ & $\mathrm{ND}$ & $\mathrm{ND}$ & $\mathrm{ND}$ & ND \\
\hline $18: 0$ & $3.25(1.25)$ & $4 \cdot 10(0 \cdot 48)$ & $4.21(1.59)$ & $4 \cdot 50(1 \cdot 22)$ & $4.99(0.72)$ & $0 \cdot 92(0 \cdot 19)$ & $2.99(1.22)$ \\
\hline 11 methyl $18: 1 \omega 7 c i s$ & $11.92(3.61)$ & $4 \cdot 82(2 \cdot 77)$ & $5 \cdot 57(4 \cdot 33)$ & $11 \cdot 14(2 \cdot 48)$ & $9.99(1.38)$ & $0.28(0.55)^{*}$ & ND \\
\hline $18: 12-\mathrm{OH}$ & ND & $0 \cdot 11(0 \cdot 32)^{*}$ & ND & $0.47(0.62)^{*}$ & ND & ND & ND \\
\hline $18: 1 \omega 5 \mathrm{cis}$ & $\mathrm{ND}$ & $\mathrm{ND}$ & $0.06(0 \cdot 21)^{*}$ & $\mathrm{ND}$ & $\mathrm{ND}$ & $\mathrm{ND}$ & $\mathrm{ND}$ \\
\hline $18: 1 \omega 9$ cis & $0 \cdot 12(0 \cdot 15)^{*}$ & ND & ND & $0.34(0 \cdot 42)^{*}$ & ND & ND & ND \\
\hline Unknown (ECL 18.794) & $0 \cdot 21(0 \cdot 38)^{*}$ & ND & $0.32(0 \cdot 49)^{*}$ & $0 \cdot 08(0 \cdot 18)^{*}$ & $0.11(0 \cdot 31)^{*}$ & ND & $0.25(0.35)^{*}$ \\
\hline $19: 0$ & ND & ND & ND & $\mathrm{ND}$ & $\mathrm{ND}$ & ND & $\mathrm{ND}$ \\
\hline $19: 0$ cyclo $\omega 8$ cis & $37 \cdot 27(8 \cdot 48)$ & $22 \cdot 96(6 \cdot 05)$ & $33.35(11.05)$ & $21.93(8 \cdot 17)$ & $12 \cdot 37(4 \cdot 31)$ & $0.57(0.48)$ & $11 \cdot 33(5 \cdot 67)$ \\
\hline 19:0 10 methyl & $0.18(0.28)^{*}$ & $1 \cdot 16(0 \cdot 45)$ & $0 \cdot 08(0 \cdot 19)^{*}$ & $1.23(0.45)$ & $0.16(0.44)^{*}$ & ND & $1.28(0.56)$ \\
\hline $20: 0$ & $0 \cdot 05(0 \cdot 11)^{*}$ & $0.04(0 \cdot 15)^{*}$ & $\mathrm{ND}$ & $0.07(0 \cdot 19)^{*}$ & $\mathrm{ND}$ & $\mathrm{ND}$ & $0.04(0 \cdot 10)^{*}$ \\
\hline $20: 1 \omega 9$ trans & $0 \cdot 14(0 \cdot 22)^{*}$ & $\mathrm{ND}$ & $0 \cdot 46(0 \cdot 40)$ & $0 \cdot 15(0 \cdot 26)^{*}$ & $\mathrm{ND}$ & $\mathrm{ND}$ & $0 \cdot 10(0 \cdot 16)^{*}$ \\
\hline $20: 2 \omega 6,9$ cis & $0.95(0.68)$ & $0.04(0 \cdot 15)^{*}$ & $0.77(0.44)$ & $0.05(0 \cdot 15)^{*}$ & ND & $\mathrm{ND}$ & ND \\
\hline $20: 3 \omega 6,9,12 c i s$ & ND & ND & $\mathrm{ND}$ & ND & ND & ND & ND \\
\hline Summed feature $2 \dagger$ & ND & ND & $\mathrm{ND}$ & ND & ND & ND & ND \\
\hline Summed feature $3 \ddagger$ & ND & ND & ND & ND & ND & ND & ND \\
\hline Summed feature $4 \S$ & $0.34(0.46)^{*}$ & $0.41(0.50)^{*}$ & $0.61(0.41)$ & $0.51(0.53)$ & $1.42(0.23)$ & $0.29(0.34)^{*}$ & $0 \cdot 44(0 \cdot 45)$ \\
\hline Summed feature $5 \|$ & ND & $\mathrm{ND}$ & $\mathrm{ND}$ & ND & ND & ND & ND \\
\hline Summed feature 7 & $24.69(8.43)$ & $42.04(7 \cdot 41)$ & $35 \cdot 81(12 \cdot 47)$ & $33 \cdot 73(6 \cdot 16)$ & $53 \cdot 22(5 \cdot 19)$ & $83.521 .52)$ & $63.02(5.67)$ \\
\hline
\end{tabular}

For footnotes, see Table 1.

iso 3-OH, 17:0 iso, 17:0 cyclo, 18:0 and 18:0 2-OH in Agrobacterium biovar 2; and 18:0, 18:1 2-OH and 19:0 10 methyl in Agrobacterium biovar 3.

These data resulted in discrete clustering of strains belonging to Agrobacterium biovar 1, Agrobacterium biovar 2 and Agrobacterium biovar 3. Comparison of the three biovars indicated that Agrobacterium biovar 1 has a fatty acid profile similar to that of Rhizobium sp. (Hedysarum coronarium), but deviates by lacking 18:0 and by containing a higher concentration of 16:0 and 16:0 3-OH. Agrobacterium biovar 2 was similar to $R$. hainanense but differed by possessing a higher concentration of 15:0 iso 3-OH, 16:0 3-OH and summed feature 4. Agrobacterium biovar 3 appeared to have a unique fatty acid composition with only a slight similarity to $R$. giardinii.

Mesorhizobium. Fatty acid compounds detected in all species of the genus Mesorhizobium included 16:0, 17:0, 17:0 iso, 18:0, 19:0 cyclo $\omega 8$ cis and summed feature 7 . Additional fatty acids detected in individual

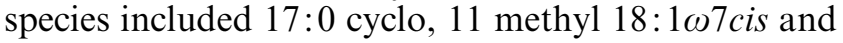
$20: 2 \omega 6,9$ cis in $M$. ciceri; $13: 0$ iso $3-\mathrm{OH}, 17: 0$ cyclo, 11

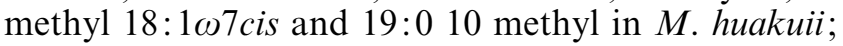

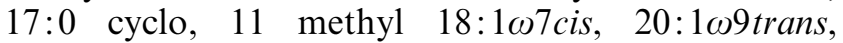

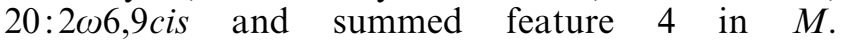
mediterraneum; 13:0 iso 3-OH, 17:0 cyclo, 11 methyl 
Table 3. Mean fatty acid concentration of Rhizobium species analysed in this study

Values are percentages of the total amount of fatty acid compounds present for that species. Numbers in parentheses indicate standard deviation.

\begin{tabular}{|c|c|c|c|c|c|c|c|c|}
\hline Fatty acid compound & R. etli & R. galegae & $\begin{array}{l}\text { Rhizobium sp. } \\
\text { (Hedysarum) }\end{array}$ & R. hainanense & R. leguminosarum & R. tropici & R. gallicum & R. giardinii \\
\hline $10: 0$ & ND & ND & ND & ND & ND & ND & ND & ND \\
\hline $10: 03-\mathrm{OH}$ & ND & ND & ND & $0 \cdot 08(0 \cdot 30)^{*}$ & ND & ND & ND & ND \\
\hline $11: 02-\mathrm{OH}$ & $0.02(0.09)^{*}$ & $\mathrm{ND}$ & ND & $\mathrm{ND}$ & $\mathrm{ND}$ & $\mathrm{ND}$ & $\mathrm{ND}$ & $\mathrm{ND}$ \\
\hline Unknown (ECL 11.541) & $\mathrm{ND}$ & ND & ND & $0 \cdot 54(0 \cdot 10)$ & ND & $0.02(0.07)^{*}$ & ND & $\mathrm{ND}$ \\
\hline Unknown (ECL 14.952) & $0 \cdot 20(0 \cdot 30)^{*}$ & $\mathrm{ND}$ & $0.02(0.07)^{*}$ & $1.09(0.19)$ & $\mathrm{ND}$ & $0.02(0.08)^{*}$ & $0 \cdot 25(0 \cdot 21)^{*}$ & $0 \cdot 21(0 \cdot 45)^{*}$ \\
\hline $12: 03-\mathrm{OH}$ & ND & ND & ND & ND & ND & ND & ND & ND \\
\hline $13: 03-\mathrm{OH}$ & ND & ND & ND & ND & ND & ND & ND & ND \\
\hline $13: 1$ at $12-13$ & ND & ND & $0 \cdot 16(0 \cdot 20)^{*}$ & $0 \cdot 85(0 \cdot 16)$ & ND & $0.07(0 \cdot 15)^{*}$ & $0 \cdot 48(0 \cdot 28)$ & ND \\
\hline $13: 0$ iso $3-\mathrm{OH}$ & ND & ND & ND & ND & ND & ND & $\mathrm{ND}$ & $\mathrm{ND}$ \\
\hline $14: 0$ & ND & ND & ND & $\mathrm{ND}$ & $\mathrm{ND}$ & ND & $\mathrm{ND}$ & $\mathrm{ND}$ \\
\hline Unknown (ECL 14·780) & ND & ND & ND & ND & ND & ND & ND & $0 \cdot 10(0 \cdot 32)^{*}$ \\
\hline $15: 0$ & ND & ND & ND & ND & ND & ND & ND & $\mathrm{ND}$ \\
\hline $15: 0$ anteiso & ND & ND & ND & ND & $0.04(0.21)^{*}$ & ND & ND & ND \\
\hline $15: 0$ iso & ND & ND & ND & ND & $0.03(0 \cdot 16)^{*}$ & $0.02(0.09)^{*}$ & $\mathrm{ND}$ & $\mathrm{ND}$ \\
\hline $15: 02-\mathrm{OH}$ & $0 \cdot 70(0 \cdot 57)$ & ND & $0.09(0.15)^{*}$ & $\mathrm{ND}$ & $0.87(0 \cdot 41)$ & ND & $0 \cdot 48(0 \cdot 16)$ & $\mathrm{ND}$ \\
\hline $15: 03-\mathrm{OH}$ & $0.03(0 \cdot 11)^{*}$ & ND & $0 \cdot 20(0 \cdot 22)^{*}$ & $\mathrm{ND}$ & $0.02(0.09)^{*}$ & $\mathrm{ND}$ & ND & ND \\
\hline $15: 0$ iso $3-\mathrm{OH}$ & ND & ND & ND & $2 \cdot 94(0 \cdot 18)$ & ND & $3.40(0.46)$ & $\mathrm{ND}$ & $\mathrm{ND}$ \\
\hline $15: 1 \omega 8$ cis & ND & ND & ND & ND & ND & ND & ND & ND \\
\hline Unknown (ECL 15.953) & ND & ND & $0.01(0.04)^{*}$ & $0.05(0 \cdot 11)^{*}$ & $\mathrm{ND}$ & $0 \cdot 11(0 \cdot 15)^{*}$ & $\mathrm{ND}$ & $\mathrm{ND}$ \\
\hline $16: 0$ & $2.53(0.46)$ & $9 \cdot 72(1 \cdot 42)$ & $4 \cdot 60(0 \cdot 58)$ & $6 \cdot 33(0 \cdot 50)$ & $4.08(1.00)$ & $7.70(1.89)$ & $3 \cdot 22(0 \cdot 42)$ & $11 \cdot 39(1.64)$ \\
\hline $16: 03-\mathrm{OH}$ & $1 \cdot 20(0 \cdot 22)$ & $3 \cdot 30(0 \cdot 29)$ & $2.64(0.20)$ & $3.53(0.31)$ & $1.32(0.28)$ & $3 \cdot 87(0 \cdot 24)$ & $2 \cdot 29(0 \cdot 17)$ & $0.03(0 \cdot 12)^{*}$ \\
\hline $16: 0$ iso & ND & ND & ND & ND & $\mathrm{ND}$ & ND & $\mathrm{ND}$ & $\mathrm{ND}$ \\
\hline $16: 1 \omega 5 \mathrm{cis}$ & ND & ND & ND & ND & ND & ND & ND & ND \\
\hline $17: 0$ & $0.32(0.50)^{*}$ & $0.43(0.41)$ & $0.99(0.51)$ & $0.33(0 \cdot 36)^{*}$ & $0.32(0.42)^{*}$ & $0.02(0 \cdot 12)^{*}$ & $0.09(0 \cdot 21)^{*}$ & $0.58(0 \cdot 47)$ \\
\hline $17: 0$ iso & ND & ND & ND & $1.32(0.33)$ & ND & $0.93(0 \cdot 27)$ & ND & ND \\
\hline $17: 0$ iso $3-\mathrm{OH}$ & ND & ND & ND & $0 \cdot 15(0 \cdot 20)^{*}$ & ND & $0.31(0.22)^{*}$ & $\mathrm{ND}$ & ND \\
\hline $17: 0$ cyclo & ND & $0 \cdot 11(0 \cdot 20)^{*}$ & $0 \cdot 48(0 \cdot 25)$ & $0 \cdot 20(0 \cdot 23)^{*}$ & $0 \cdot 15(0 \cdot 28)^{*}$ & $0.68(0 \cdot 42)$ & $0.02(0 \cdot 09)^{*}$ & $1 \cdot 77(0 \cdot 71)$ \\
\hline $17: 03-\mathrm{OH}$ & $\mathrm{ND}$ & $0.09(0 \cdot 19)^{*}$ & $0.06(0 \cdot 11)^{*}$ & ND & $\mathrm{ND}$ & ND & $\mathrm{ND}$ & ND \\
\hline $17: 1 \omega 6 \mathrm{cis}$ & ND & ND & ND & ND & ND & ND & ND & ND \\
\hline $17: 1 \omega 7 c i s$ & $\mathrm{ND}$ & ND & $\mathrm{ND}$ & $\mathrm{ND}$ & $\mathrm{ND}$ & $\mathrm{ND}$ & $\mathrm{ND}$ & $\mathrm{ND}$ \\
\hline $17: 1 \omega 8$ cis & $0.03(0 \cdot 11)^{*}$ & $0.06(0 \cdot 15)^{*}$ & $0.24(0.23)^{*}$ & $\mathrm{ND}$ & $0.01(0.07)^{*}$ & $\mathrm{ND}$ & ND & $0 \cdot 12(0 \cdot 25)^{*}$ \\
\hline Unknown (ECL 17.875) & ND & ND & ND & ND & ND & ND & ND & ND \\
\hline $18: 0$ & $8 \cdot 71(1 \cdot 21)$ & $1 \cdot 20(0 \cdot 32)$ & $5.75(1.04)$ & $3.98(0.60)$ & $7.83(1.97)$ & $2 \cdot 87(1 \cdot 21)$ & $9 \cdot 26(2 \cdot 41)$ & $2 \cdot 35(0 \cdot 67)$ \\
\hline 11 methyl $18: 1 \omega 7 c i s$ & $1.48(2.02)$ & $0 \cdot 10(0 \cdot 19)^{*}$ & $0.02(0.07)^{*}$ & $0.89(0.37)$ & $4 \cdot 70(2 \cdot 65)$ & $0.55(0.24)$ & ND & $2 \cdot 87(1.35)$ \\
\hline $18: 12-\mathrm{OH}$ & ND & ND & ND & $1.95(0.60)$ & $0 \cdot 05(0 \cdot 24)^{*}$ & $0.82(0.66)$ & ND & $\mathrm{ND}$ \\
\hline $18: 1 \omega 5 \mathrm{cis}$ & $\mathrm{ND}$ & $\mathrm{ND}$ & $\mathrm{ND}$ & $\mathrm{ND}$ & $\mathrm{ND}$ & ND & $\mathrm{ND}$ & ND \\
\hline $18: 1 \omega 9$ cis & $0 \cdot 12(0 \cdot 30)^{*}$ & ND & $0.05(0.08)^{*}$ & ND & $0.09(0.21)^{*}$ & ND & $0.03(0.06)^{*}$ & $\mathrm{ND}$ \\
\hline Unknown (ECL 18.794) & $0.06(0 \cdot 19)^{*}$ & $0.02(0.07)^{*}$ & $0 \cdot 11(0 \cdot 15)^{*}$ & $\mathrm{ND}$ & $0 \cdot 03(0 \cdot 11)^{*}$ & $\mathrm{ND}$ & $\mathrm{ND}$ & $\mathrm{ND}$ \\
\hline $19: 0$ & ND & ND & ND & ND & $0.01(0.06)^{*}$ & ND & ND & ND \\
\hline $19: 0$ cyclo $\omega 8$ cis & $10 \cdot 19(5 \cdot 44)$ & $31 \cdot 12(9 \cdot 31)$ & $20 \cdot 00(8 \cdot 13)$ & $38 \cdot 84(4 \cdot 27)$ & $13.91(5.32)$ & $49 \cdot 29(9 \cdot 76)$ & $7 \cdot 30(3 \cdot 49)$ & $2 \cdot 78(1 \cdot 36)$ \\
\hline 19:0 10 methyl & $0.01(0.06)^{*}$ & $2.32(0.36)$ & $0 \cdot 21(0.17)^{*}$ & ND & $0.02(0.12)^{*}$ & ND & $0 \cdot 10(0 \cdot 16)^{*}$ & $1.53(0.33)$ \\
\hline $20: 0$ & $\mathrm{ND}$ & ND & ND & $\mathrm{ND}$ & $\mathrm{ND}$ & $\mathrm{ND}$ & $\mathrm{ND}$ & ND \\
\hline $20: 1 \omega 9$ trans & $0.09(0 \cdot 16)^{*}$ & ND & ND & ND & $0.01(0.05)^{*}$ & $\mathrm{ND}$ & $0 \cdot 23(0 \cdot 18)^{*}$ & ND \\
\hline $20: 2 \omega 6,9$ cis & $\mathrm{ND}$ & $0 \cdot 11(0 \cdot 17)^{*}$ & $0.05(0.12)^{*}$ & $0.57(0.13)$ & ND & $0.39(0 \cdot 22)$ & $\mathrm{ND}$ & $\mathrm{ND}$ \\
\hline $20: 3 \omega 6,9,12 c i s$ & $1.95(0 \cdot 37)$ & $1.25(0 \cdot 19)$ & $0.87(0.06)$ & $2 \cdot 80(0 \cdot 42)$ & $2 \cdot 31(0.53)$ & $1.83(0.65)$ & $0 \cdot 77(0 \cdot 22)$ & $2.66(0.49)$ \\
\hline Summed feature $2 \dagger$ & ND & ND & ND & ND & ND & ND & $\mathrm{ND}$ & $\mathrm{ND}$ \\
\hline Summed feature $3 \ddagger$ & $5.95(0 \cdot 68)$ & $4.82(0.39)$ & $6.42(0.63)$ & $3 \cdot 30(0 \cdot 32)$ & $6 \cdot 46(0 \cdot 94)$ & $2 \cdot 33(0 \cdot 67)$ & $6 \cdot 25(0 \cdot 43)$ & $10 \cdot 45(2 \cdot 13)$ \\
\hline Summed feature $4 \S$ & $0 \cdot 22(0 \cdot 24)^{*}$ & $0.57(0.32)$ & $1.06(0.21)$ & $0.09(0 \cdot 17)^{*}$ & $0.52(0.35)$ & $0 \cdot 16(0 \cdot 23)^{*}$ & $0.58(0 \cdot 19)$ & $4.37(0.95)$ \\
\hline Summed feature $5 \|$ & ND & $\mathrm{ND}$ & ND & ND & ND & ND & ND & ND \\
\hline Summed feature 7 & $66 \cdot 17(8 \cdot 01)$ & $44 \cdot 78(10 \cdot 75)$ & $55.99(8.18)$ & $30 \cdot 16(5 \cdot 16)$ & $57 \cdot 22(6 \cdot 49)$ & $24 \cdot 61(11 \cdot 00)$ & $68.65(2.65)$ & $58.80(4 \cdot 62)$ \\
\hline
\end{tabular}

For footnotes, see Table 1.

18: $1 \omega 7 c i s, 19: 010$ methyl and summed feature 4 in $M$. loti; 11 methyl 18: $1 \omega 7 \mathrm{cis}$ and summed feature 4 in $M$. tianshanense group I; and 13:0 iso $3-\mathrm{OH}, 17: 1 \omega 8 \mathrm{cis}$, 19:0 10 methyl and summed feature 4 in $M$. plurifarium. This genus could be distinguished from the other genera because it lacked 20:3 $\omega 6,9,12 \mathrm{cis}$ and summed feature 3 and possessed 17:0 iso fatty acids.

$M$. ciceri possessed a unique fatty acid profile with similarities to $M$. mediterraneum. It differed from other species belonging to this genus by containing a lower concentration of summed feature 7 and a higher concentration of 19:0 cyclo $\omega 8$ cis. This organism separated from $M$. mediterraneum due to its higher concentration of the 11 methyl 18:167cis. $M$. mediterraneum appeared to be similar to $M$. ciceri, but could be distinguished from the other species based on the concentration of summed feature 7 and 19:0 cyclo $\omega 8$ cis. Analysis of $M$. tianshanense strains revealed two groups. These groups were designated $M$. tianshanense fatty acid groups I and II. Group I appeared to belong to the genus Mesorhizobium with similarities to the ' $M$. ciceri-M. mediterraneum' cluster. Unlike the other members of this genus, this group contained much less of 19:0 cyclo $\omega 8$ cis and a greater concentration of summed feature 7. Group II of M. tianshanense 
Table 4. Mean fatty acid concentration of Sinorhizobium species analysed in this study

Values are percentages of the total amount of fatty acid compounds present for that species. Numbers in parentheses indicate standard deviation.

\begin{tabular}{|c|c|c|c|c|c|c|}
\hline Fatty acid compound & S. fredii & S. meliloti & S. saheli & S. terangae & S. kostiense & S. arboris \\
\hline $10: 0$ & ND & ND & ND & ND & ND & ND \\
\hline $10: 03-\mathrm{OH}$ & ND & ND & ND & ND & ND & ND \\
\hline $11: 02-\mathrm{OH}$ & ND & ND & ND & ND & ND & ND \\
\hline Unknown (ECL 11.541) & ND & ND & ND & ND & ND & ND \\
\hline Unknown (ECL 14.952) & ND & ND & $0 \cdot 26(0 \cdot 44)^{*}$ & $0 \cdot 15(0 \cdot 25)^{*}$ & $0 \cdot 37(0 \cdot 80)^{*}$ & $0 \cdot 13(0 \cdot 39)^{*}$ \\
\hline $12: 03-\mathrm{OH}$ & ND & ND & ND & ND & ND & ND \\
\hline $13: 03-\mathrm{OH}$ & ND & ND & ND & ND & ND & ND \\
\hline $13: 1$ at $12-13$ & ND & ND & ND & ND & ND & ND \\
\hline $13: 0$ iso $3-\mathrm{OH}$ & ND & ND & ND & ND & ND & ND \\
\hline $14: 0$ & ND & ND & ND & ND & ND & ND \\
\hline Unknown (ECL 14.780) & ND & ND & ND & ND & $0.06(0 \cdot 19)^{*}$ & ND \\
\hline $15: 0$ & ND & ND & ND & ND & ND & ND \\
\hline $15: 0$ anteiso & $0 \cdot 10(0 \cdot 44)^{*}$ & ND & ND & ND & ND & ND \\
\hline $15: 0$ iso & $0.01(0.06)^{*}$ & ND & ND & ND & ND & ND \\
\hline $15: 02-\mathrm{OH}$ & ND & ND & ND & ND & ND & ND \\
\hline $15: 03-\mathrm{OH}$ & ND & ND & ND & ND & ND & ND \\
\hline $15: 0$ iso $3-\mathrm{OH}$ & ND & ND & ND & ND & ND & ND \\
\hline $15: 1 \omega 8$ cis & ND & ND & ND & ND & $\mathrm{ND}$ & $\mathrm{ND}$ \\
\hline Unknown (ECL 15.953) & ND & ND & ND & ND & ND & ND \\
\hline $16: 0$ & $5 \cdot 12(1 \cdot 34)$ & $4.93(1 \cdot 14)$ & $5 \cdot 01(1 \cdot 34)$ & $3 \cdot 30(0 \cdot 93)$ & $4.94(1 \cdot 17)$ & $5 \cdot 20(1.08)$ \\
\hline $16: 03-\mathrm{OH}$ & $0.49(0 \cdot 29)$ & $0 \cdot 68(0 \cdot 12)$ & $0.61(0.52)^{*}$ & $0 \cdot 18(0 \cdot 24)^{*}$ & $0.03(0 \cdot 15)^{*}$ & $0 \cdot 10(0 \cdot 29)^{*}$ \\
\hline $16: 0$ iso & $0.03(0.13)^{*}$ & ND & ND & ND & ND & ND \\
\hline $16: 1 \omega 5 \mathrm{cis}$ & ND & ND & ND & ND & ND & ND \\
\hline $17: 0$ & $0 \cdot 73(0 \cdot 46)$ & $0 \cdot 54(0 \cdot 24)$ & $0 \cdot 08(0 \cdot 20)^{*}$ & $0 \cdot 24(0 \cdot 29)^{*}$ & $0.56(0.60)$ & $\mathrm{ND}$ \\
\hline $17: 0$ iso & ND & ND & ND & ND & ND & ND \\
\hline $17: 0$ iso $3-\mathrm{OH}$ & ND & ND & ND & ND & ND & ND \\
\hline 17:0 cyclo & $0 \cdot 20(0 \cdot 35)^{*}$ & $0 \cdot 75(0 \cdot 31)$ & $0 \cdot 15(0 \cdot 36)^{*}$ & ND & $0 \cdot 04(0 \cdot 16)^{*}$ & ND \\
\hline $17: 03-\mathrm{OH}$ & ND & $0 \cdot 05(0 \cdot 10)^{*}$ & ND & $0.02(0.06)^{*}$ & ND & ND \\
\hline $17: 1 \omega 6$ cis & ND & ND & ND & ND & ND & ND \\
\hline $17: 1 \omega 7 c i s$ & ND & ND & $\mathrm{ND}$ & ND & ND & ND \\
\hline $17: 1 \omega 8$ cis & $0 \cdot 11(0 \cdot 16)^{*}$ & $0.09(0 \cdot 14)^{*}$ & $\mathrm{ND}$ & $0.03(0.08)^{*}$ & ND & ND \\
\hline Unknown (ECL 17.875) & ND & ND & ND & ND & ND & ND \\
\hline $18: 0$ & $4.99(1 \cdot 15)$ & $2.69(0 \cdot 73)$ & $1.08(0.77)$ & $3.44(0.87)$ & $4 \cdot 02(1 \cdot 12)$ & $3.91(0.63)$ \\
\hline 11 methyl $18: 1 \omega 7 c i s$ & ND & $0.02(0.07)^{*}$ & ND & ND & ND & ND \\
\hline $18: 12-\mathrm{OH}$ & ND & ND & ND & ND & ND & ND \\
\hline $18: 1 \omega 5 \mathrm{cis}$ & ND & ND & ND & ND & ND & ND \\
\hline $18: 1 \omega 9$ cis & $0 \cdot 11(0 \cdot 18)^{*}$ & $0 \cdot 09(0 \cdot 07)^{*}$ & ND & $0.01(0.03)^{*}$ & $0 \cdot 05(0 \cdot 14)^{*}$ & ND \\
\hline Unknown (ECL 18.794) & $0 \cdot 19(0 \cdot 19)^{*}$ & $0 \cdot 11(0 \cdot 16)^{*}$ & ND & $0 \cdot 05(0 \cdot 13)^{*}$ & ND & ND \\
\hline $19: 0$ & ND & ND & ND & ND & ND & ND \\
\hline $19: 0$ cyclo $\omega 8$ cis & $4 \cdot 37(3.63)$ & $22 \cdot 53(8 \cdot 24)$ & $3 \cdot 55(2 \cdot 25)$ & $2 \cdot 92(2 \cdot 00)$ & $4 \cdot 02(2 \cdot 18)$ & $8 \cdot 94(4 \cdot 99)$ \\
\hline 19:0 10 methyl & $0.41(0.32)$ & $0 \cdot 85(0 \cdot 26)$ & $0.80(0.65)$ & $0.07(0 \cdot 16)^{*}$ & $0.67(0.51)$ & ND \\
\hline $20: 0$ & ND & ND & $\mathrm{ND}$ & ND & ND & ND \\
\hline $20: 1 \omega 9$ trans & $0 \cdot 12(0 \cdot 26)^{*}$ & $\mathrm{ND}$ & ND & ND & ND & $\mathrm{ND}$ \\
\hline $20: 2 \omega 6,9 c i s$ & ND & $0.02(0.07)^{*}$ & ND & ND & ND & ND \\
\hline $20: 3 \omega 6,9,12 c i s$ & $3.28(0.92)$ & $2 \cdot 94(0 \cdot 34)$ & $3.74(1.06)$ & $2.00(0.53)$ & $2 \cdot 98(0 \cdot 68)$ & $3.43(0 \cdot 37)$ \\
\hline Summed feature $2 \dagger$ & ND & ND & ND & $0.01(0.05)^{*}$ & $0.09(0.22)^{*}$ & ND \\
\hline Summed feature $3 末$ & $7 \cdot 62(2 \cdot 26)$ & $6 \cdot 44(0 \cdot 70)$ & $13 \cdot 57(3 \cdot 31)$ & $7 \cdot 70(2 \cdot 28)$ & $10 \cdot 34(1.87)$ & $9 \cdot 71(2 \cdot 73)$ \\
\hline Summed feature $4 \S$ & $0.63(0.37)$ & $0.34(0.21)$ & $1.24(0.33)$ & $0 \cdot 42(0 \cdot 34)$ & $0.50(0 \cdot 45)$ & $0.14(0.41)^{*}$ \\
\hline Summed feature $5 \|$ & ND & ND & ND & $0 \cdot 08(0 \cdot 32)^{*}$ & ND & ND \\
\hline Summed feature 79 & $71 \cdot 45(5 \cdot 25)$ & $56.93(8.25)$ & $69 \cdot 91(4 \cdot 50)$ & $79 \cdot 40(3 \cdot 27)$ & $71 \cdot 31(4 \cdot 31)$ & $68 \cdot 44(8 \cdot 16)$ \\
\hline
\end{tabular}

For footnotes, see Table 1.

appeared to belong to the genus Bradyrhizobium and possessed an almost identical fatty acid composition to Bradyrhizobium sp. (Arachis hypogaea). M. plurifarium was similar to $M$. tianshanense fatty acid group I, but contained a lower concentration of 17 iso and 18:0. It differed from other members of the genus because it contained the highest concentration of summed feature 7 and lacked 11 methyl 18:1 $107 \mathrm{cis}$, which was detected in all other species of the genus Mesorhizobium at levels greater than 4\%. The concentration of 19:0 cyclo $\omega 8$ cis was lower than the other species of the genus, but comparable to $M$. tianshanense fatty acid group I.
Rhizobium. Fatty acids detected in all species of the genus included 16:0, 18:0, 19:0 cyclo $\omega 8$ cis, $20: 3 \omega 6,9,12 c i s$, summed feature 3 and summed feature 7. Additional fatty acids detected in individual species included unknown (ECL 11.541) and unknown (ECL 14.952), $13: 1$ at $12-13,15: 0$ iso $3-\mathrm{OH}, 16: 03-\mathrm{OH}$,

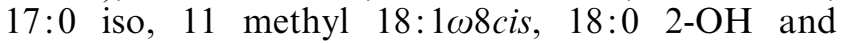
$20: 2 \omega 6,9$ cis in $R$. hainanense; 17:0, 17:0 cyclo, 11 methyl 18:1 $\omega 8 \mathrm{cis}, 19: 010$ methyl and summed feature 4 in $R$. giardinii; $13: 1$ at $12-13,15: 02-\mathrm{OH}, 16: 03-\mathrm{OH}$ and summed feature 4 in $R$. gallicum; $15: 0$ iso $3-\mathrm{OH}$,

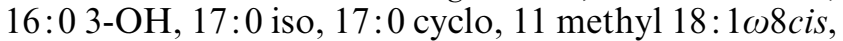

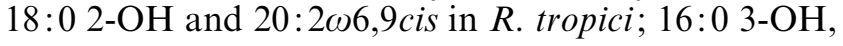


17:0, 19:0 10 methyl and summed feature 4 in $R$. galegae; 16:0 3-OH, 17:0, 17:0 cyclo and summed feature 4 in Rhizobium sp. (Hedysarum coronarium); 15:0 2-OH, 16:0 3-OH, 11 methyl 18:168cis and summed feature 4 in $R$. leguminosarum; and 15:0 2-

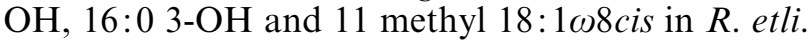

The fatty acid profile of Rhizobium sp. (Hedysarum coronarium) was similar to that of $S$. meliloti and $R$. leguminosarum; however, it deviated by containing significantly higher levels of 16:0 3-OH. It could be further distinguished from $S$. meliloti and other members of the genus Rhizobium by containing the lowest levels of $20: 3 \omega 6,9,12 \mathrm{cis}$ and a moderate concentration of 19:0 cyclo $\omega 8$ cis. $R$. hainanense was similar to R. tropici and Agrobacterium biovar 2 in a separate lineage containing 15:0 iso $3-\mathrm{OH}$ which is unique to this group. Although $R$. hainanense was similar to Agrobacterium biovar 2, it could be distinguished by the presence of 13:1, unknown (ECL 14.952) and unknown (ECL 11.541). It was notable that $R$. hainanense, $R$. tropici and Agrobacterium biovar 2 were the only species that contained 15:0 iso 3-OH. Furthermore, R. hainanense, Agrobacterium biovar 2, Agrobacterium biovar 3 and R. tropici all contained 18:1 2-OH at similar levels. R. gallicum appeared to be most similar to $R$. etli, but could be distinguished by containing a higher concentration of 16:0 3-OH and a lower concentration of $20: 3 \omega 6,9,12$ cis. Further discrimination was possible by the presence of 11 methyl 18:167cis in R. etli and not in $R$. gallicum. Analysis of $R$. giardinii revealed significant differences from the other rhizobia. It contained a higher concentration of 16:0, 17:0 cyclo, summed feature 3 and summed feature 4 and the lowest concentration of 19:0 cyclo $\omega 5$ cis among the rhizobia. It is further distinguished by lacking 16:0 3-OH, a significant fatty acid which contributes to distinguishing the genera Rhizobium and Sinorhizobium.

Sinorhizobium. Fatty acids common to all species of this genus included 16:0, 18:0, 19:0 cyclo $\omega 8 \mathrm{cis}$, $20: 3 \omega 6,9,12 \mathrm{cis}$, summed feature 3 and summed feature 7. Additional fatty acids detected in individual species included 16:0 3-OH, 17:0, 17:0 cyclo, 19:0 10 methyl and summed feature 4 in $S$. meliloti; 16:0 3$\mathrm{OH}, 17: 0,19: 010$ methyl and summed feature 4 in $S$. fredii; 17:0, 19:0 10 methyl and summed feature 4 in $S$. kostiense; 19:0 10 methyl and summed feature 4 in $S$. saheli; and summed feature 4 in $S$. terangae.

Fatty acid patterns in the genus Sinorhizobium were similar to those of the genus Rhizobium, but differed by generally containing a greater concentration of summed feature 7 and a lower concentration of 19:0 cyclo $\omega 8$ cis and 16:03-OH. Although this relationship holds for most of the species, $S$. meliloti has concentrations of summed feature 7 and 19:0 cyclo $\omega 8 \mathrm{cis}$ more characteristic of the genus Rhizobium, but contains 16:0 3-OH at a concentration characteristic of the genus Sinorhizobium. This suggests that 16:0 3-
$\mathrm{OH}$ is an important fatty acid for discriminating between the genera.

Results indicated that fatty acid analysis is in agreement with phylogenetic data that place $S$. fredii into this genus. $S$. saheli was similar to $S$. fredii but differed because it contained a higher concentration of summed feature 3 and a lower concentration of 18:0. Since only six strains were available for analysis, conclusive results were not obtainable. Analysis of $S$. terangae revealed a close similarity to $S$. fredii and $S$. saheli, with only minor differences in the concentration of summed feature 7. S. kostiense, S. arboris and S. fredii appeared to be very similar to one another with insignificant differences in the concentration of 17:0, 19:0 10 methyl and 19:0 cyclo w8cis. Data collected for this genus revealed a higher level of fatty acid similarity between species than for the other genera evaluated.

Bradyrhizobium. The fatty acids detected in all species of the genus Bradyrhizobium were 16:0 and summed feature 7 . Additional fatty acids detected in individual species included 16:1 $\omega 5 \mathrm{cis}, 17: 1 \omega 8 \mathrm{cis}, 18: 0,11$ methyl 18: $1 \omega 7$ cis, summed feature 4 and $19: 0$ cyclo $\omega 8$ cis in

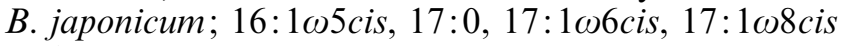
and 19:0 cyclo $\omega 8 \mathrm{cis}$ in B. liaoningense; 17:0 cyclo, 18:0, 19:0 cyclo $\omega 8$ cis and summed feature 4 in B. elkanii group I; 19:0 cyclo $\omega 8$ cis and summed feature 4 in $B$. elkanii group II; and 16:1 $105 \mathrm{cis}$ in Bradyrhizobium sp. (Arachis hypogaea).

Comparisons of bradyrhizobia fatty acid profiles with those of other members of the genera indicate that they possess a unique ratio of fatty acids. Unlike other rhizobia, bradyrhizobia are composed primarily of 16:0 and summed feature 7 that make up $90 \%$ of the total fatty acids. Examination of B. elkanii indicated that two subgroups exist which were distinguished by the concentration of 19:0 cyclo $\omega 8 \mathrm{cis}$ and summed feature 7 . These two groups were designated B. elkanii fatty acid groups I and II for the purposes of this manuscript. This finding has also been reported elsewhere (Graham et al., 1995). B. elkanii could be distinguished from $B$. japonicum by containing a greater concentration of $19: 0$ cyclo $\omega 8$ cis. $B$. liaoningense strains could be distinguished from other bradyrhizobia by containing a greater concentration of 16:0 and possessing a concentration of 19:0 cyclo $\omega 8$ cis and summed feature 7 between that of B. elkanii and B. japonicum. Strains of Bradyrhizobium sp. (Arachis hypogaea) were similar to B. japonicum, but differed by containing a greater concentration of 16: $1 \omega 5$ cis and insignificant levels of 19:0 cyclo $\omega 8$ cis.

\section{Similarity matrix}

The data presented in Table 5 indicate the relative similarity between species as determined by the Sherlock MIS cluster analysis software. Values presented in the similarity matrix are computed on the basis of the identification values of all strains. Identi- 
Table 5. Relative percentage similarity between species and groups based on identification indices derived from MIS software

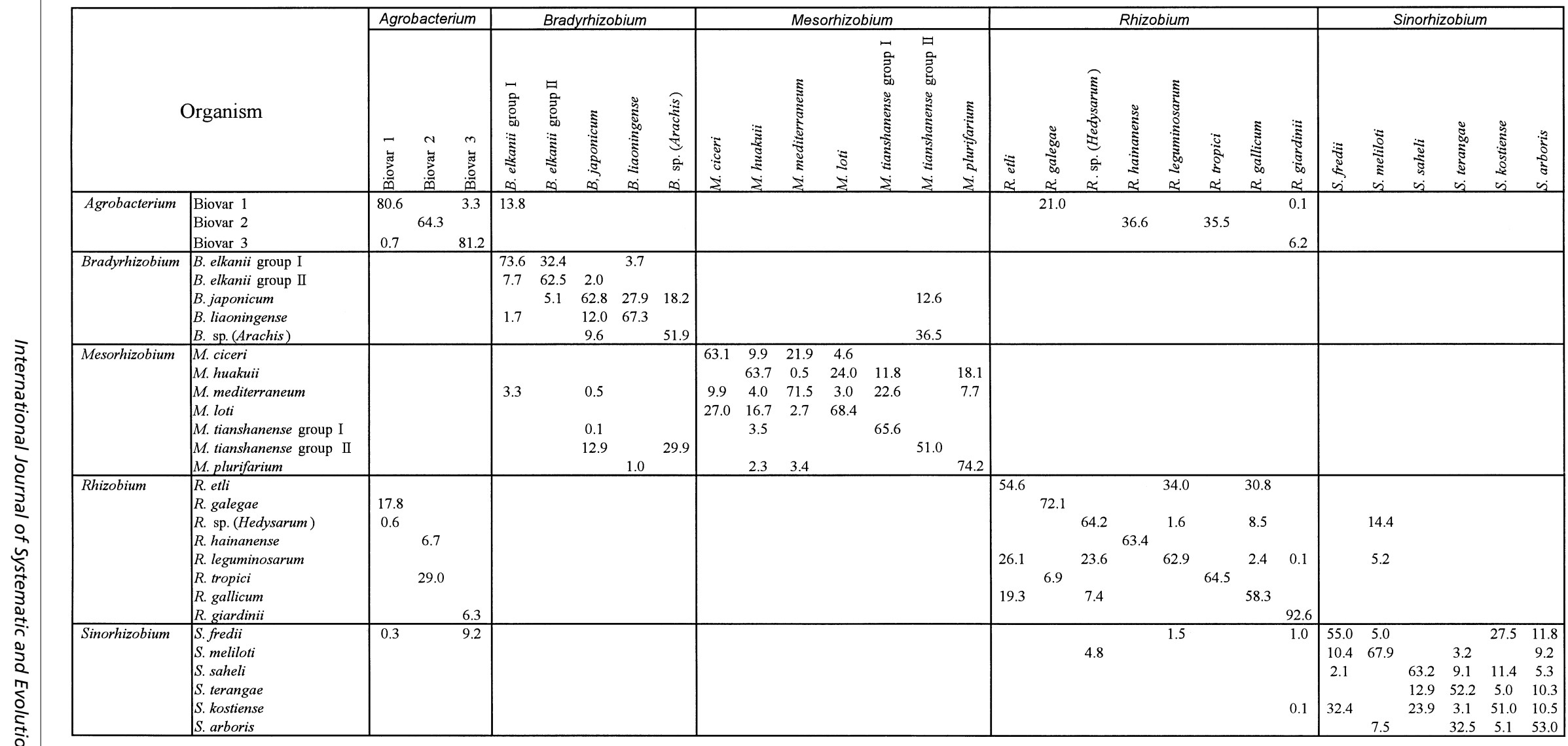


fication values are not included in this manuscript due to the large quantity of data they encompass. Proper evaluation of the table is done by inspecting in a vertical fashion.

Our data suggested that a group with a similarity value of less than $55 \%$ to itself is difficult or impossible to distinguish from the next most similar group using this method. When this value was observed in conjunction with a value of $27 \%$ or greater to another group, it was not possible to discriminate the groups. However, it was notable that discrepancies in values could be observed for species of which less than twenty strains were analysed. An example of this can be observed when comparing the similarity values of $M$. ciceri $(9$ strains examined) with $M$. loti (17 strains examined). When comparing the vertical column value for $M$. ciceri to the horizontal value of $M$. loti, a value of $27 \%$ is observed, but when comparing the vertical column value of $M$. loti to the horizontal value of $M$. ciceri, a $4.6 \%$ similarity is observed. This unfortunate situation is a shortcoming of the MIS software when not enough strains of each species are used to build a species fatty acid profile. Although this situation could have been avoided by evaluating more strains, they were not available when this study was conducted.

Similarity values for biovars belonging to the genus Agrobacterium indicated that Agrobacterium biovar 1 has similarity to R. galegae, and Agrobacterium biovar 2 has similarity to R. tropici. Agrobacterium biovar 3 has low similarity with Agrobacterium biovar 1, $R$. giardinii and $S$. fredii. Similarity values for members of the genus Bradyrhizobium indicate that B. elkanii fatty acid group I has similarity to Agrobacterium biovar 1, $B$. elkanii fatty acid group II and $B$. liaoningense. $B$. elkanii fatty acid group II has a high similarity to $B$. elkanii fatty acid group I and low similarity to $B$. japonicum. $B$. liaoningense has similarity to $B$. japonicum and little similarity with $B$. elkanii and M. plurifarium. Bradyrhizobium. sp. (Arachis hypogaea) has a strong similarity to $M$. tianshanense fatty acid group II and B. japonicum. Interestingly, the similarity between Bradyrhizobium. sp. (Arachis hypogaea) and $M$. tianshanense fatty acid group II is so high that these organisms are undistinguishable. Further evaluation is needed to support the classification of strains belonging to $M$. tianshanense fatty acid group II.

Similarity values for species belonging to the genus Mesorhizobium indicate that $M$. ciceri is similar to $M$. loti and less similar to $M$. mediterraneum. Likewise, $M$. mediterraneum shows similarity with $M$. ciceri and little similarity with $M$. loti and $M$. plurifarium. $M$. tianshanense fatty acid group I shows similarity to $M$. mediterraneum and less to $M$. huakuii. M. plurifarium appears to be unique, with similarity to $M$. huakuii and M. mediterraneum.

Results for the genus Rhizobium indicated a high similarity between $R$. etli and R. leguminosarum. This is not unexpected since it is known that these species are closely related (Segovia et al., 1993). Rhizobium sp. (Hedysarum coronarium) has a similarity with $R$. leguminosarum and $S$. meliloti, and less with $R$. gallicum. $R$. hainanense has similarity with Agrobacterium biovar 2, whilst $R$. gallicum has similarity with $R$. etli and less with Rhizobium sp. (Hedysarum coronarium) and $R$. leguminosarum. $R$. giardinii has little similarity with Agrobacterium biovar 3 and appears to be unique.

Similarities among the sinorhizobia appeared to be greater than for other genera. These data showed that $S$. fredii is similar to $S$. kostiense and S. meliloti. $S$. meliloti has similarity with both Rhizobium sp. (Hedysarum coronarium) and S. arboris. S. saheli has similarity with both $S$. terangae and $S$. kostiense, whilst $S$. terangae is most similar to $S$. arboris. S. kostiense has similarity with $S$. fredii and the two are indistinguishable using this method. Similarity values for $S$. arboris seem to be evenly weighted for all the other species belonging to this genus.

\section{3-D principal component plot}

The 3-D principal component plot shown in Fig. 1 was assembled from coordinates generated by from 2-D principal component data of the Sherlock MIS software. This type of plot is very useful when evaluating species relatedness based on clustering. Although this plot appears to reveal the separation of many of the groups, it should be noted that the principal components are composed of a combination of several fatty acids and do not take into account all fatty acids. Unfortunately, the selection of fatty acids used in the multivariate calculations to generate the plot are determined by the MIS software and cannot be modified by the investigator without changes to the source code. Although the 3-D plot does not take into account all fatty acids detected, it is effective at clustering similar groups and separating groups that exhibit a significant degree of dissimilarity.

This plot indicates that the species belonging to the genus Agrobacterium are distributed throughout the Agrobacterium-Rhizobium-Sinorhizobium supercluster. The position of Agrobacterium biovar 1 is separate from other groups and related to $R$. galegae. Agrobacterium biovar 2 is close to $R$. hainanense and $R$. tropici, whilst Agrobacterium biovar 3 clusters near $R$. giardinii, $R$. fredii, $S$. saheli and $S$. arboris. The genus Mesorhizobium is separate in this plot from other genera and is composed of three clusters that contain $M$. ciceri and $M$. mediterraneum, $M$. tianshanense fatty acid group I and M. plurifarium, and $M$. huakuii and M. loti. Clustering of species belonging to the genus Rhizobium indicates that $R$. etli, $R$. gallicum, $R$. leguminosarum and Rhizobium $\mathrm{sp}$. (Hedysarum coronarium) make up a single cluster. The positions of $R$. hainanense and $R$. tropici are near that of Agrobacterium biovar 2, whilst $R$. galegae clusters 


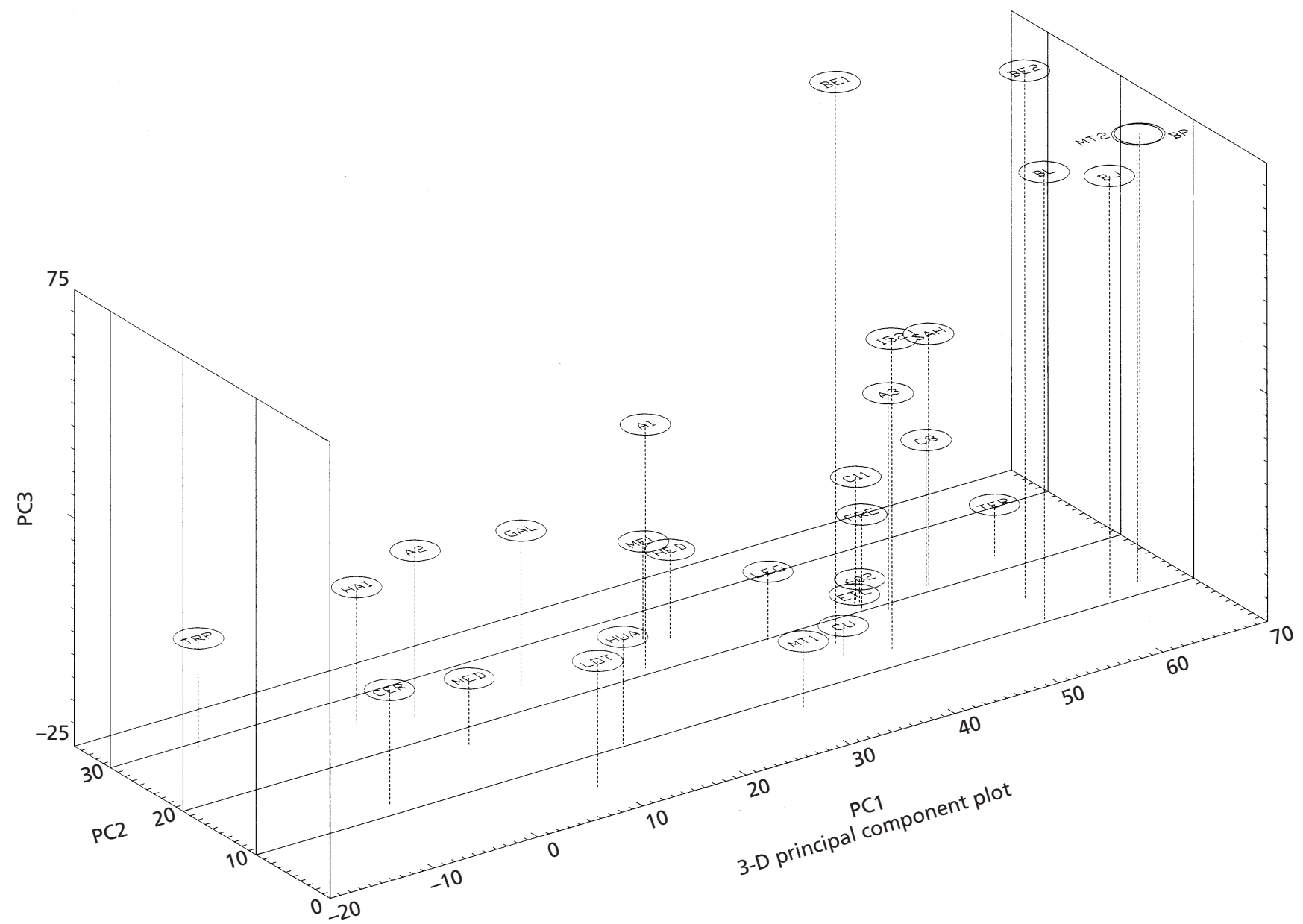

Fig. 1. 3-D plot of fatty acid principal components for all species evaluated in this study. A1, Agrobacterium biovar $1 ; A 2$, Agrobacterium biovar 2; A3, Agrobacterium biovar 3; BE1, Bradyrhizobium elkanii group I; BE2, Bradyrhizobium elkanii group II; BL, Bradyrhizobium liaoningense; BP, Bradyrhizobium sp. (Arachis); CER, Mesorhizobium ciceri; HUA, Mesorhizobium huakuii; LOT, Mesorhizobium loti; MED, Mesorhizobium mediterraneum; MT1, Mesorhizobium tianshanense group I; MT2, Mesorhizobium tianshanense group II; CU, Mesorhizobium plurifarium; ETL, Rhizobium etli; GAL, Rhizobium galegae; HED, Rhizobium sp. (Hedysarum); HAl, Rhizobium hainanense; LEG, Rhizobium leguminosarum; TRP, Rhizobium tropici; 602, Rhizobium gallicum; 152, Rhizobium giardinii; FRE, Sinorhizobium fredii; MEL, Sinorhizobium meliloti; SAH, Sinorhizobium saheli; TER, Sinorhizobium terangae; C8, Sinorhizobium kostiense; C11, Sinorhizobium arboris.

near Agrobacterium biovar 1 and Agrobacterium biovar 2 . The position of $R$. giardinii is unique, but appears to cluster near Agrobacterium biovar 3. In the genus Sinorhizobium, S. meliloti clusters near Rhizobium sp. (Hedysarum coronarium), S. fredii clusters with $S$. arboris, $R$. etli and $R$. gallicum, and $S$. saheli clusters with $S$. kostiense and $R$. giardinii. The position of $S$. terangae appears separate from other members of the genus.

The position of the genus Bradyrhizobium on this plot is quite remote compared to the other genera. This is not surprising considering that the genus Bradyrhizobium is vastly different from the other rhizobia. In this plot, B. japonicum and B. elkanii fatty acid types I and II are separate, but the positions of B. liaoningense,
Bradyrhizobium sp. (Arachis hypogaea) and $R$. tianshanense group II are close to B. japonicum.

\section{DISCUSSION}

In this study, the Sherlock Microbial Identification System (MIS) was used to collect cellular fatty acid data and to evaluate relationships between species belonging to the genera Agrobacterium, Bradyrhizobium, Mesorhizobium, Rhizobium and Sinorhizobium. Although previous studies had examined several species belonging to these genera, they did not involve such a large number of strains from so many species as here. In this study, we have included some previous data which have been amended to include the 
compound 11 methyl 18:167cis, which had been previously detected but unidentified. This additional piece of data slightly modifies data reported in our previous studies (Jarvis et al., 1996; Jarvis \& Tighe, 1994). This discovery affects the total quantity of fatty acids in each strain and the overall relationships between strains. Considering the new compounds and using the data collected, we have assembled a table of fatty acids, a similarity matrix and a 3-D principal component plot for all the species examined.

Results collected for the genus Agrobacterium revealed three clusters corresponding to Agrobacterium biovar 1, Agrobacterium biovar 2 and Agrobacterium biovar 3. In agreement with phylogenetic data, Agrobacterium biovar 1 shows similarity to $R$. galegae (Terefework et al., 1998) whilst Agrobacterium biovar 2 has similarity to $R$. tropici and $R$. hainanense (Chen et al., 1997). Agrobacterium biovar 3 has a unique ratio of fatty acids with very little similarity to the genus Rhizobium. Data for $A$. rubi (DNA group TR2) were not included in this study because only two strains were available for analysis.

Evaluation of the genus Bradyrhizobium revealed significant fatty acid differences from all other rhizobia studied. This is not surprising as research has shown that bradyrhizobia are significantly different from the other rhizobia and agrobacteria (Jordan, 1982). Our results indicate that B. japonicum and B. elkanii are readily distinguished from one another, and that two fatty acid subgroups of $B$. elkanii were observed. $B$. liaoningense has been described as an extra slowgrowing (ESG) species with similarities to B. japonicum (Xu et al., 1995). This is supported by cellular fatty acid data and only minor differences in two fatty acids have been observed between the two species. Strains of Bradyrhizobium sp. (Arachis hypogaea) were also similar to B. japonicum but because of such similar fatty acid profiles, reliable discrimination was not possible.

The genus Mesorhizobium was established to accommodate organisms belonging to the $R$. loti-R. huakuii cluster (Jarvis et al., 1997). Fatty acid analysis of this genus showed clear discrimination between the species. The phylogenetically defined species evaluated in this study include $M$. huakuii, $M$. loti, $M$. ciceri, $M$. mediterraneum, $M$. tianshanense and $M$. plurifarium. Although fatty acid analysis has previously been performed on several of the species belonging to this group, an important discriminating fatty acid has since been identified as 11 methyl 18:1 107 cis and included in this study. This compound is useful for discriminating between species belonging to the genus Mesorhizobium and for discrimination from other genera.

The similarities observed between $M$. ciceri, M. loti and $M$. mediterraneum support the phylogenetic data reported between these species (Jarvis et al., 1997). $M$. huakuii strains formed a separate cluster with similarity to the $M$. loti cluster. $M$. plurifarium is similar to $M$. tianshanense fatty acid group I and less similar to $M$. loti. Although this dissimilarity is not evident in the similarity matrix or 3-D plot, it can be detected when evaluating the fatty acid data in Table 1 . This finding supports other studies that define $M$. plurifarium as a new species (de Lajudie et al., 1998b).

Analysis of strains received as $M$. tianshanense revealed two distinct groups with different fatty acid profiles. These have been designated fatty acid groups I and II for the purposes of this investigation. Fatty acid group I appears similar to $M$. huakuii and M. plurifarium and these data support the earlier designation of these strains as a new species belonging to the genus Mesorhizobium based on phylogenetic studies (Chen et al., 1995; Jarvis et al., 1997). The four strains constituting fatty acid group II appear identical to Bradyrhizobium sp. (Arachis hypogaea) and could not be distinguished using fatty acid analysis. This suggests that the original classification of these strains may be questionable.

Although the fatty acid characteristics and relationships of the genus Rhizobium have been previously described, their relationship with new species belonging to the genus Sinorhizobium has not been addressed. The clustering of rhizobial species based on fatty acid analysis is unusual because several separate clusters are observed. R. etli, $R$. gallicum, Rhizobium sp. (Hedysarum coronarium) and $R$. leguminosarum form one cluster. Within this cluster, $R$. etli with $R$. leguminosarum and $R$. gallicum form a subcluster with closer fatty acid similarity. The fatty acid profiles of these species permit effective discrimination between $R$. gallicum and R. etli, but are less able to discriminate between R. leguminosarum and R. etli.

Fatty acid analysis suggests that Rhizobium sp. (Hedysarum coronarium) has a unique fatty acid pattern with close similarity to $S$. meliloti. However, these two organisms can be distinguished based on the concentration of $20: 3 \omega 6,9,12 \mathrm{cis}$ and 16:0 3-OH. $R$. hainanense, $R$. tropici and $R$. galegae form a separate fatty acid cluster with comparatively little similarity to other species belonging to the genus Rhizobium. Fatty acid data collected for $R$. hainanense, $R$. tropici and Agrobacterium biovar 2 are in broad agreement with phylogenetic data which indicates similarity of the species (Lindström et al., 1995). Nonetheless, fatty acid analysis is capable of discriminating between the species. Phylogenetic data indicate that $R$. galegae and $R$. giardinii have similarities to members of the genus Agrobacterium (Amarger et al., 1997). These reports support our findings that indicate $R$. galegae is similar to Agrobacterium biovar 1 , and $R$. giardinii has little similarity to Agrobacterium biovar 3 . In both cases, fatty acid analysis is able to discriminate between these species.

The genus Sinorhizobium was established in 1988 to accommodate the fast-growing soybean rhizobia (Chen et al., 1988). Currently this genus contains eight named species, of which we have examined six. The similarities observed among species belonging to this 
genus using fatty acid analysis are much greater than those seen in other genera evaluated. Interestingly, our results indicate a discriminatory role for the fatty acid 16:0 3-OH. Organisms belonging to the genus Sinorhizobium contain low levels of this compound, whilst members of the genus Rhizobium contain significantly higher levels. This suggests that 16:0 $3-\mathrm{OH}$ is important for discriminating between the two genera and can be observed in the case of S. meliloti and Rhizobium sp. (Hedysarum coronarium). These two organisms possess a very similar fatty acid profile and cluster together in the 3-D plot, but the concentration of 16:0 3-OH serves to place them in their respective genera.

Although the fatty acid data we have collected for the genera Agrobacterium, Rhizobium and Bradyrhizobium can be compared with that of other researchers (Yokota \& Sakane, 1991; Yokota et al., 1993), they evaluated fewer strains and species and used different strains, growth media and analytical methods to detect the fatty acids. This resulted in fatty acid data that was compositionally different from that observed in this study using the Sherlock MIS. However, the data collected by both groups are in broad agreement.

Although the sole use of fatty acid data to compare one species with another is not typically employed for the purposes of taxonomy, it can be used in conjunction with other taxonomic information such as phylogenetic data. Similarities derived from fatty acid analysis are in broad agreement with $16 \mathrm{~S}$ rDNA results and appear to accurately distinguish between most species. The Sherlock MIS is an attractive tool in phenotypic taxonomy because it can rapidly assay a large number of unknown strains and correlates well with genomic data.

\section{ACKNOWLEDGEMENTS}

Funding was provided by in-kind contributions of the authors and their affiliations. Provision of strains by the following persons is gratefully acknowledged: N. Amarger, Centre de Microbiologie des Sols et de l'Environnement, Dijon Cedex, France; A. Squartini, Dipartimento di Biotecnologie Agrarie, Universita Degli Studi di Padova, Italy; W. X. Chen, J. L. Gao and E. T. Wang, Department of Microbiology, Beijing Agricultural University, Beijing, Peoples' Republic of China; B. D. Eardly, Pennsylvania State University, Berks Campus, Reading, PA, USA; M. Fenton, Department of Microbiology and Genetics, Massey University, Palmerston North, New Zealand; D. Jenssens, LMG Culture Collection, Laboratorium voor Microbiologie, K. L. Ledeganckstraat 35, Belgium; M. E. Martinez-Romero, Centro de Investigacion sobre Fijacion de Nitrogeno, Universidad Nacional Autonoma de Mexico, Mexico; J. P. W. Young, Department of Biology, University of York, Heslington, Yorkshire, UK; M. Fernandez, Ecologie Microbienne des Sols, Universite Claude BernardLyon I, Villeurbanne Cedex, France; L. M. Xu, Soils and Fertilizers Institute, Caas Beijing, Peoples' Republic of China; P. H. Graham, Soil Science Department, University of Minnesota, St Paul, MN, USA; X. Zhang, Department of Applied Microbiology, Sichuan Agriculture University, Sichuan, Peoples' Republic of China; Z. Xiang-hong,
Huazhong Agricultural University, Wuhan, Peoples' Republic of China.

\section{REFERENCES}

Amarger, N., Macheret, V. \& Laguerre, G. (1997). Rhizobium gallicum sp. nov. and Rhizobium giardinii sp. nov. from Phaseolus vulgaris nodules. Int J Syst Bacteriol 47, 996-1006.

van Berkum, P., Beyene, D., Bao, G., Campbell, A. \& Eardly, B. D. (1998). Rhizobium mongolense sp. nov. is one of three rhizobial genotypes identified which nodulate and form nitrogen-fixing symbioses with Medicago ruthenica [(L.) Ledebour]. Int J Syst Bacteriol 48, 13-22.

Bradbury, J. F. (1986). Agrobacterium. In A Guide to Plant Pathogenic Bacteria, pp. 6-15. Farnham Royal: CAB International Mycological Institute.

Casella, S., Shapleigh, J.P. \& Payne, W. J. (1986). Nitrate reduction in "Rhizobium hedysari" strain HCNT 1. Arch Microbiol 146, 233-238.

Chen, W. X., Yan, G. H. \& Li, J. L. (1988). Numerical taxonomic study of fast-growing soybean rhizobia and a proposal that Rhizobium fredii be assigned to Sinorhizobium gen. nov. Int $J$ Syst Bacteriol 38, 392-397.

Chen, W. X., Wang, E. T., Li, Y. B., Chen, X. Q. \& Li, Y. (1995). Characteristics of Rhizobium tianshanense sp. nov., a moderately and slowly growing root nodule bacterium isolated from an arid saline environment in Xinjiang, People's Republic of China. Int J Syst Bacteriol 45, 153-159.

Chen, W. X., Tan, Z. Y., Gao, J. L., Li, Y. \& Wang, E. T. (1997). Rhizobium hainanense sp. nov., isolated from tropical legumes. Int J Syst Bacteriol 47, 870-873.

Graham, P. H., Sadowsky, M. J., Tighe, S. W., Thompson, J. A., Date, R. A., Howieson, J. G. \& Thomson, R. (1995). Differences among strains of Bradyrhizobium in fatty acid-methyl ester analysis. Can J Microbiol 41, 1038-1042.

Jarvis, B. D. W. \& Tighe, S. W. (1994). Rapid identification of Rhizobium species based on cellular fatty acid analysis. Plant Soil 161, 31-41.

Jarvis, B. D. W., Downer, H. L. \& Young, J. P. W. (1992). Phylogeny of fast-growing soybean-nodulating rhizobia supports synonymy of Sinorhizobium and Rhizobium and assignment to Rhizobium fredii. Int J Syst Bacteriol 42, 93-96.

Jarvis, B. D. W., Sivakumaran, S., Tighe, S. W. \& Gillis, M. (1996). Identification of Agrobacterium and Rhizobium species based on cellular fatty acid composition. Plant Soil 184, 143-158.

Jarvis, B. D. W., Van Berkum, P., Chen, W. X., Nour, S. M., Fernandez, M. P., Cleyet-Marel, J. C. \& Gillis, M. (1997). Transfer of Rhizobium huakuii, Rhizobium ciceri, Rhizobium mediterraneum, and Rhizobium tianshanense to Mesorhizobium gen. nov. Int J Syst Bacteriol 47, 895-898.

Jordan, D. C. (1982). Transfer of Rhizobium japonicum Buchanan 1980 to Bradyrhizobium gen. nov., a genus of slow-growing root nodule bacteria from leguminous plants. Int J Syst Bacteriol 32, 136-139.

Jordan, D. C. (1984). Family III Rhizobiaceae. Bergey's Manual of Systematic Bacteriology, vol. 1, pp. 234-244. Edited by N. R. Krieg \& J. G. Holt. Baltimore: Williams \& Wilkins.

Keyser, H. H., Bohlool, B. B., Hu, B. \& Weber, D. (1982). Fast growing rhizobia isolated from root nodules of soybean. Science 215, 1631-1632.

de Lajudie, P., Willems, A., Pot, B. \& 7 other authors (1994). Polyphasic taxonomy of rhizobia: emendation of the genus 
Sinorhizobium and description of Sinorhizobium meliloti comb. nov., Sinorhizobium saheli sp. nov. and Sinorhizobium terangae sp. nov. Int J Syst Bacteriol 44, 715-733.

de Lajudie, P., Laurent-Fulele, E., Willems, A., Torck, U., Coopman, R., Collins, M. D., Kersters, K., Dreyfus, B. \& Gillis, M. (1998a). Allorhizobium undicola gen. nov., sp. nov., nitrogen-fixing bacteria that efficiently nodulate Neptunia natans in Senegal. Int J Syst Bacteriol 48, 1277-1290.

de Lajudie, P., Willems, A., Nick, G. \& 9 other authors (1998b). Characterization of tropical tree rhizobia and description of Mesorhizobium plurifarium sp. nov. Int J Syst Bacteriol 48, 369-382.

Lindström, K., Van Berkum, P., Gillis, M., Martinez, E., Novikova, N. \& Jarvis, B. D. W. (1995). Nitrogen Fixation: Fundamentals and Applications. Report from the Roundtable on Rhizobium Taxonomy, pp. 807-810. Edited by I. A. Tikhonovich, N. A. Provorov, V. I. Romanov \& W. E. Newton. Dordrecht: Kluwer.

Long, S. (1989). Rhizobium-legume nodulation: life together in the underground. Cell 56, 203-214.

Lynch, J. M. (1976). Products of soil microorganisms in relation to plant growth. Crit Rev Microbiol 5, 67-107.

Nick, G., de Lajudie, P., Eardly, B. D., Suomalainen, S., Paulin, L., Zhang, X., Gillis, M. \& Lindström, K. (1999). Sinorhizobium arboris sp. nov. and Sinorhizobium kostiense sp. nov., isolated from leguminous trees in Sudan and Kenya. Int J Syst Bacteriol 49, 1359-1368.

Orgambide, G. G., Reusch, R. N. \& Dazzo, F. B. (1993). Methoxylated fatty acids reported in Rhizobium isolates arise from chemical alterations of common fatty acids upon acidcatalyzed transesterification procedures. $J$ Bacteriol $\mathbf{1 7 5}$, 4922-4926.

Rome, S., Fernandez, M. P., Brunel, B., Normand, P. \& CleyetMarel, J. C. (1996). Sinorhizobium medicae sp. nov., isolated from annual Medicago spp. Int J Syst Bacteriol 46, 972-980.

Sasser, M. (1990). Identification of bacteria by gas chromatography of cellular fatty acids. MIDI Technical Note 101. Microbial ID, Inc., Newark, DE, USA.
Sawada, H., Takikawa, Y. \& leki, H. (1992). Fatty acid methyl ester profiles of the genus Agrobacterium. Ann Phytopathol Soc Jpn 58, 46-51.

Segovia, L., Young, J. P. W. \& Martinez-Romero, E. (1993). Reclassification of American Rhizobium leguminosarum biovar phaseoli type I strains as Rhizobium etli sp. nov. Int J Syst Bacteriol 43, 374-377.

Terefework, Z., Nick, G., Suomalainen, S., Paulin, L. \& Lindström, K. (1998). Phylogeny of Rhizobium galegae with respect to other rhizobia and agrobacteria. Int J Syst Bacteriol 48, 349-356.

Wang, E. T., Van Berkum, P., Beyene, D., Sui, X. H., Chen, W. X. \& Martinez-Romero, E. (1998). Rhizobium huautlense sp. nov., a symbiont of Sesbania herbacea that has a close relationship with Rhizobium galegae. Int J Syst Bacteriol 48, 687-699.

Wang, E. T., Van Berkum, P., Sui, X. H., Beyene, D., Chen, W. X. \& Martinez-Romero, E. (1999). Diversity of rhizobia associated with Amorpha fruticosa isolated from Chinese soils and description of Mesorhizobium amorphae sp. nov. Int $J$ Syst Bacteriol 49, 51-65.

Willems, A. \& Collins, M. D. (1993). Phylogenetic analysis of rhizobia and agrobacteria based on $16 \mathrm{~S}$ ribosomal DNA sequences. Int J Syst Bacteriol 43, 305-313.

Xu, L. M., Ge, C., Cui, Z., Li, J. \& Fan, H. (1995). Bradyrhizobium liaoningense sp. nov., isolated from the root nodules of soybeans. Int J Syst Bacteriol 45, 706-711.

Yokota, A. \& Sakane, T. (1991). Taxonomic significance of fatty acid compositions in whole cells and lipopolysaccharides in Rhizobiaceae. Inst Ferment Res Commun 15, 57-75.

Yokota, A., Sakane, T., Ophel, K. \& Sawada, H. (1993). Further studies on the cell fatty acid composition of Rhizobium and Agrobacterium species. Inst Ferment Res Commun 16, 86-94.

Young, J. P. W. \& Haukka, K. E. (1996). Diversity and phylogeny of rhizobia. New Phytol 133, 87-94.

Zhang, X., Nick, G., Kaijalainen, S., Terefework, Z., Paulin, L., Tighe, S. W., Graham, P. H. \& Lindström, K. (1999). Phylogeny and diversity of Bradyrhizobium strains isolated from the root nodules of peanut (Arachis hypogaea) in Sichuan, China. Syst Appl Microbiol 22, 378-386. 